CONF-890379--3

DE89 013041

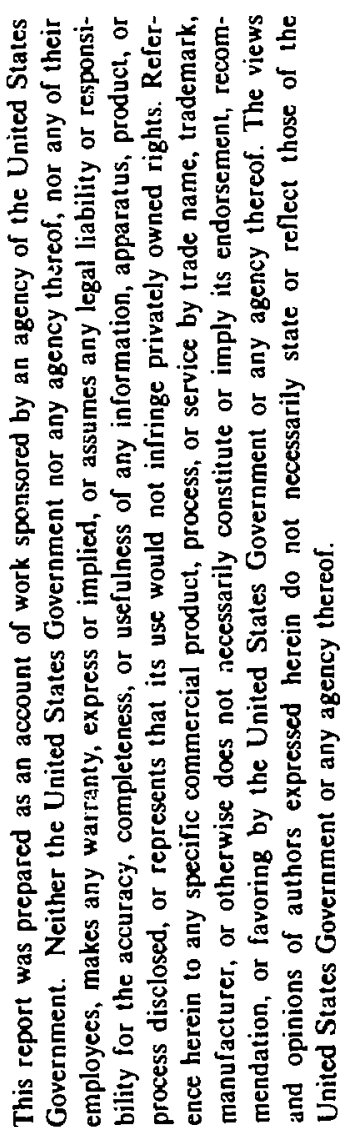

\title{
Detectors for the Superconducting Super Collider, Design Concepts, and Simulation*
}

T. A. Gabriel

Oak Ridge National Laboratory

Engineering Physics and Mathematics Divisicn

P.O. Box 2008, MS-6364

Oak Ridge, TN 37831-6364

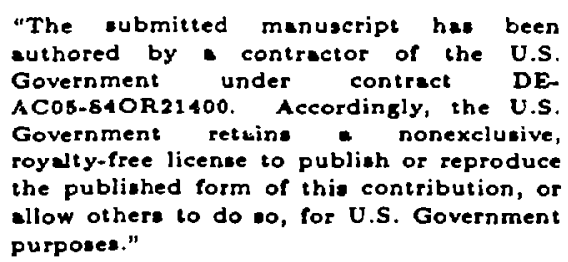

Paper presented at Workshop on Calorimetry for the Superconducting Super Collider, University of Alabama, Tuscalcosa, AL, March 13-17, 1989.

* Research sponsored by Office of High Energy and Nuclear Physics, U.S. Department of Energy under contract number DE-AC05-84OR21400 with Martin Marietta Energy Systems, Inc.

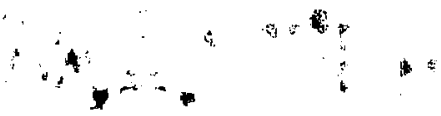




\title{
Detectors for the Superconducting Super Collider Design Concepts, and Simulation
}

\author{
T. A. Gabriel \\ Oak Ridge National Laboratory \\ Engineering Physics and Mathematics Division \\ P.O. box 2008, MS-6364 \\ Oak Ridge, TN 37831-6364
}

\begin{abstract}
The phy'sics of compensation calorimetry is reviewed in the light of the needs of the Superconducting Super Collider (SSC) detectors. The four major detector types: liquid argon, scintillator, room temperature liquids, and silicon, are analyzed with respect to some of their strengths and weaknesses. Finally, general comments are presented which reflect the reliability of simulation code systems.
\end{abstract}

\section{INTRODUCTION}

During the next several years difficult decisions must be made concerning the types of calorimeters to be used in the main SSC detectors. To insure the best choice, a working knowledge of the physics of compensating calorimetry and of the advantages and disadvantages of the major calorimeter type must be known and recognized by a large fraction of the high energy physics community. To this end, the topics to be covered in this paper include summarizing some of the important factors determining calorimeter performance, compensation, and resolution, as well as surveying some of the detectors that are being considered for the SSC, including their advantages and disadvantages, resolution, and compensating abilities. The detector types considered are liquid argon, scintillator, room temperature liquids, and silicon.

Since a large amount of design data is generated from simulation programs, a short discussion will also be given concerning the amount of confidence that can be put in these code systems. These cornments will center around the CALOR89 system since the author has the most familiarity with these programs.

\section{FUNDAMENTAL PHYSICS OF COMPENSATING CALORIMETERS}

\subsection{Basic Ideas}

Before one is able to assess the prospects for hadron calorimetry with any of the active materials being considered (liquid argon, scintillator, room temperature liquids, and silicon), it is essential to understand the details of 
compensating calorimetry. This begins with an appreciation for the original motivation of the inventors of the technicue. 1] It must be realized that hadronic cascades which contain large amounts of electromagnetic energy have less losses of energy due to nuclear break-up than those which contain little electromagnetic energy and therefore a large number of hadronic interactions. This comparison is illustrated in Fig. 1. Figure la shows the situation for noncompensating calorimeters. The overall distribution is created by summing a variety of different types of events. The two extremes, here called type $A$ and type $B$. result in quite different responses. The type $A$ events have a large fraction of the incident hadron energy converted to $\pi^{0}$ and other forms of electromagnetic energy, and therefore there are few hadronic nuclear interactions. Since the $\pi^{0}$ yield an observed signal which is comparable to the response of the calorimeter to incident electrons of the same energy and there is little energy lost to the breakup of nuclei, the e/h ratio for such events is nearly one, as shown in Fig. 1a. Type B events in such noncompensating calorimeters, on the other hand, show large amounts of lost energy in the nuclear break up resulting from the many hadronic nuclear interactions. This includes nuclear binding energy losses, as well as losses from heavy fragment production, neutrino production, and low energy nucleon generation. All of these processes result in a reduction in the detectable energy in the calorimeter. These type $B$ cascades have a minimal amount of generated electromagnetic energy and the resulting $\mathrm{e}_{/} / \mathrm{h}$ ratio is much greater than one. Combining all types of hadronic cascades, from type A to type B and everything in between, leads to a very broad distribution of responses, and a resulting poor resolution for the measured energy deposition.

Figure $1 \mathrm{~b}$ illustrates the situation for compensating calorimeters where the response to events of type $A$ and type $B$ have been equalized by a cleverly designed calorimeter. This is the basis for the original ideas as presented in Ref. 1. It can be seen from the figure that type $A$ events respond with a signal nearly equal to an electron signal. However, the type $B$ events also give an average response that is nearly comparable to the response of an electron. The nuclear breakup energy has been compensated for, by designing the calorimeter to preferentially respond to the low energy neutron component of the shower, which is correlated with the lost nuclear breakup energy. As will be shown later, it is not necessary to fully recover all of the nuclear breakup energy as the electromagnetic response is also suppressed in high $\mathrm{Z}$ materials due to processes occurring at the very low energy portion of the electromagetic shower. The role of these sampling inefficiencies which result from the preferential photon absorption $^{2]}$ and the electron multiple scattering in the inactive material ${ }^{3,4}$ ] has a very stong effect on the calorimeter response. A proper balance of the electromagnetic suppression and the preferential sampling of low energy neutrons leads to this equalization of the average response of type $A$ and type $B$ events. As a result, the sum of all the types of events, from $A$ to $B$, yields a narrower distribution; that is, the energy resolution is greatly improved. This is the motivation for examining the ratio $e / h$ as a measure of the degree of compensation. 


\section{REPRODUCED FROM BEST
AVAILABLE COPY}
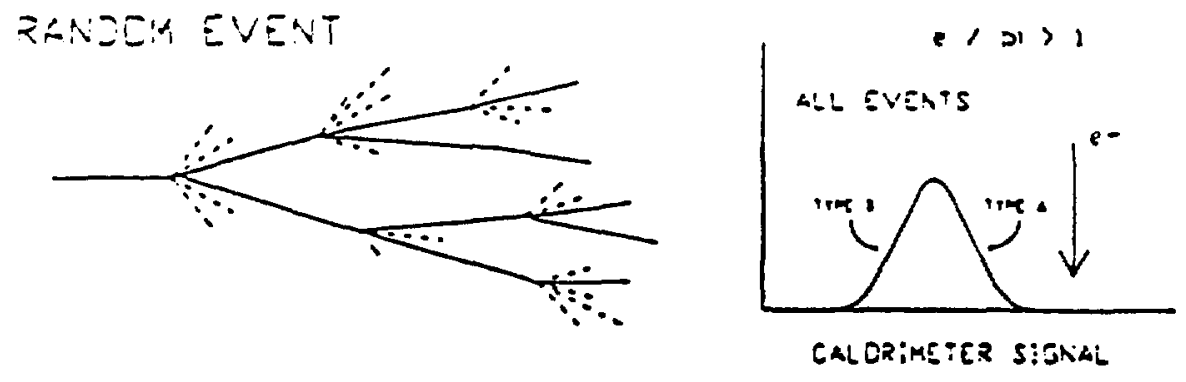

EYTRENE EVENT: TYPE A 'Ima!! $8 E$ :Lss mastiy Em eneray

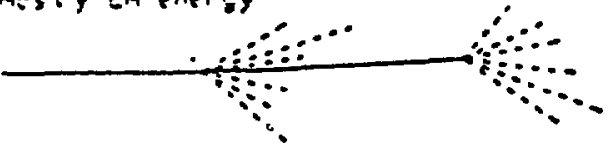

\section{EXTREME EVENT: TYPE 3}

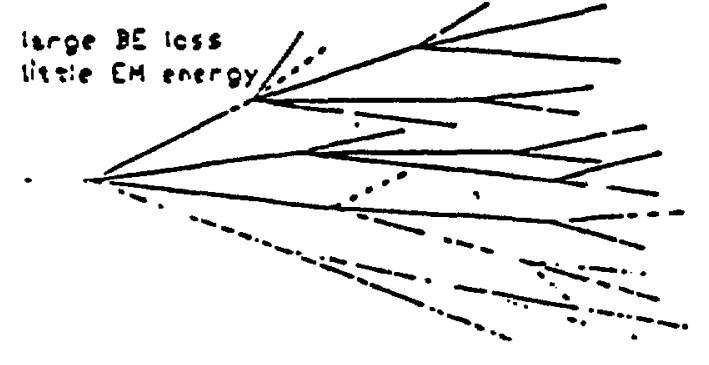

TYPE A EVENTS

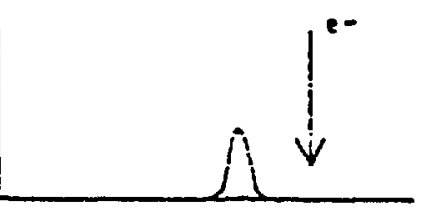

CALDRIMETER SIENAL

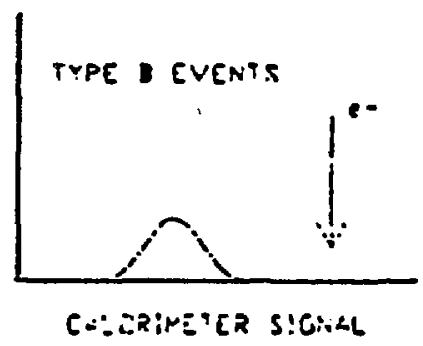

Fig. (1a). An illustration of the origin of $e / h>1$ in noncompensating calorimeters. 


\section{REPRODUCED FROM BEST AVAILABLE COPY}

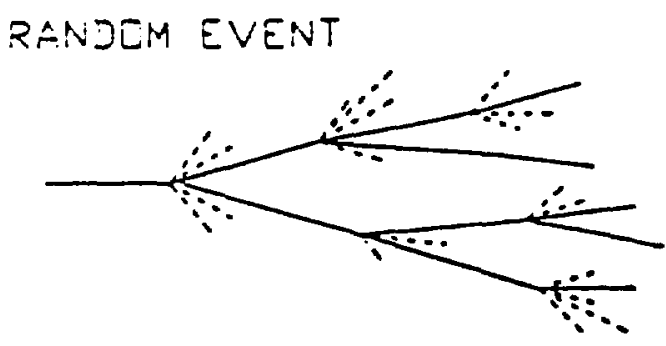

EXTREME EVENT: TYPE A 'smoll' BE ioss

nestiy EM energy

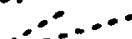

$\because: \div$

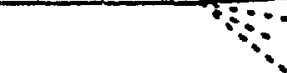

(n)
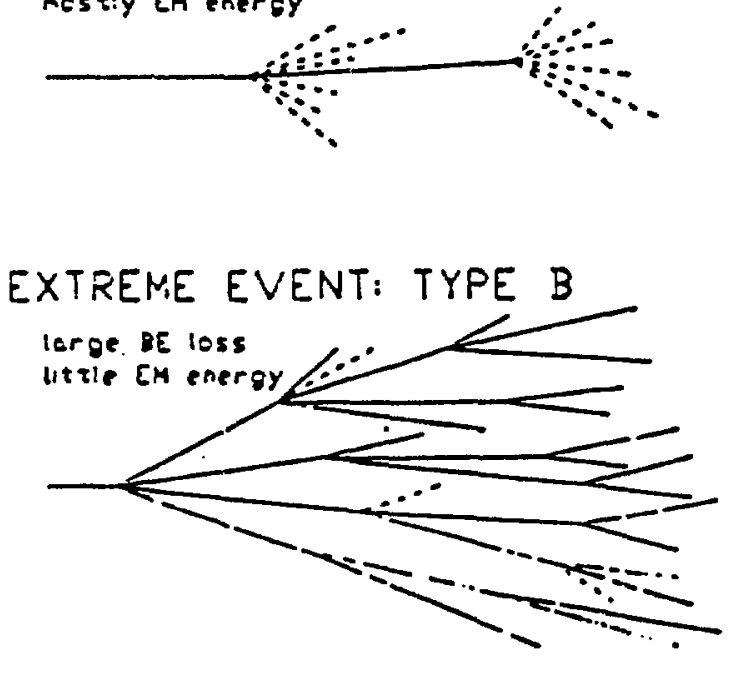

$13-1$

ALL EVENTS

acitce resaution

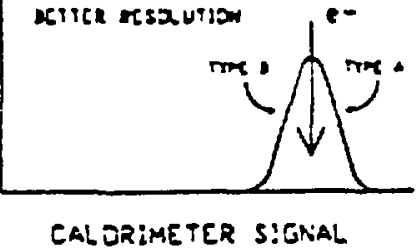

CALSRIMETER SIGNAL

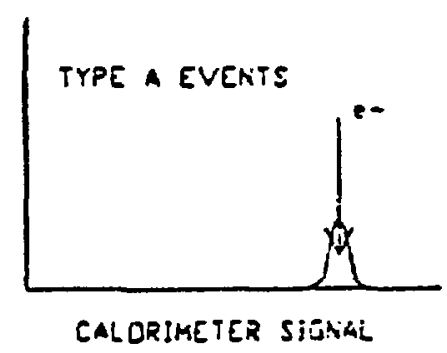

TYPE E EVENTS

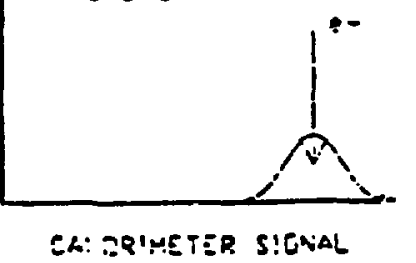

Fig. (1b). An illustration of the origin of $e / h \approx 1$ in compensating calorimeters. 


\subsection{Binding Energy Losses}

In previous studies of the detailed processes occuring in hadronic cascades, extensive use has been made of the CALOR code system (HETC, EGS, MORSE, MICAP, SPECT, LIGHT, etc.). ${ }^{5,6]}$ Figure 2 presents the calculated energy production and loss percentages due to various energy protons in an infinite uranium block. The charged hadronic (including muons) particles have been transported in HETC to yield curve B. However, curves $A$ (electromagnetic) and D (low energy neutrons) represent the amount of produced energy. The binding energy curve has been obtained from an energy balance.

On a verage at $5 \mathrm{GeT} .35 \%$ of the incident kinetic energy is lost in binding energy associated with the ejection of nucleons (primarily low energy) from the U nucleus. A total of $1750 \mathrm{MeV}$ has been lost in the form of binding energy, but one remaining effect is the large number of low energy neutrons created in the nuclear disruption. Figure 3 show's the correlation of the energy content in the low energy spallation and evaporation neutrons (kinetic energy below 20 $\mathrm{MeV}$ ) with the binding energy lost in each of the hadronic cascades. Events with small binding energy losses naturally have disrupted the nuclei to the smallest extent and have transferred the least energy to low energy nucleons. Erents with large binding energy losses have resulted from a large number of very disruptive collisions, resulting in large amounts of energy being carried by the low energy nucleons. These trends are very evident in Figure 3. It was this strong correlation which motivated the first attempts at the construction of compensating calorimeters. ${ }^{1]}$ It was reasoned that by increasing the neutron content through the fission process in the calorimeter, the binding energy losses could be overcome in a fashion which was proportional to the losses, thereby leading to an improved resolution. To achieve this end, uranium was employed as a radiator material.

\subsection{Neutrons and Hydrogenous Media}

The key to successfully capitalizing on this correlation presented in Fig 3 is through the coupling of these low energy neutrons to the sampling medium. Eventually, most of these low energy neutrons are captured by ${ }^{238} \mathrm{U}$, yielding approximately eight $\mathrm{MeV}$ per neutron capture in prompt gammas. In addition, prompt fission gammas are produced in the fission process. Unfortunately, the sampling medium is very inefficient to sampling these soft gammas due to the effects mentioned earlier regarding preferential absorption in the high $Z$ material. Also the time scale associated with thermalization of the neutrons is too long $(\sim \mu \mathrm{sec})$. Therefore, the signal from the neutrons must come from their direct interaction in the active medium. Figure 4 shows the observed neutron energy which calculations predict will be sampled by the plastic scintillator in the three millimeter uranium-scintillator stack. This distribution results from detailed calculations which were performed with the Oak Ridge Monte Carlo Codes HETC and MORSE. The solid line drawn on this figure represents the average energy carried by the spallation neutrons before transport; this is the same line as is shown on Fig. 3. Notice that a sizeable fraction of the original neutron energy (even after the effects of saturation have been taken into account $)$ has been observed $(\sim 12 \%)$. This is due to neutron amplification by fission of the uranium and the very efficient transfer of neutron energy to 


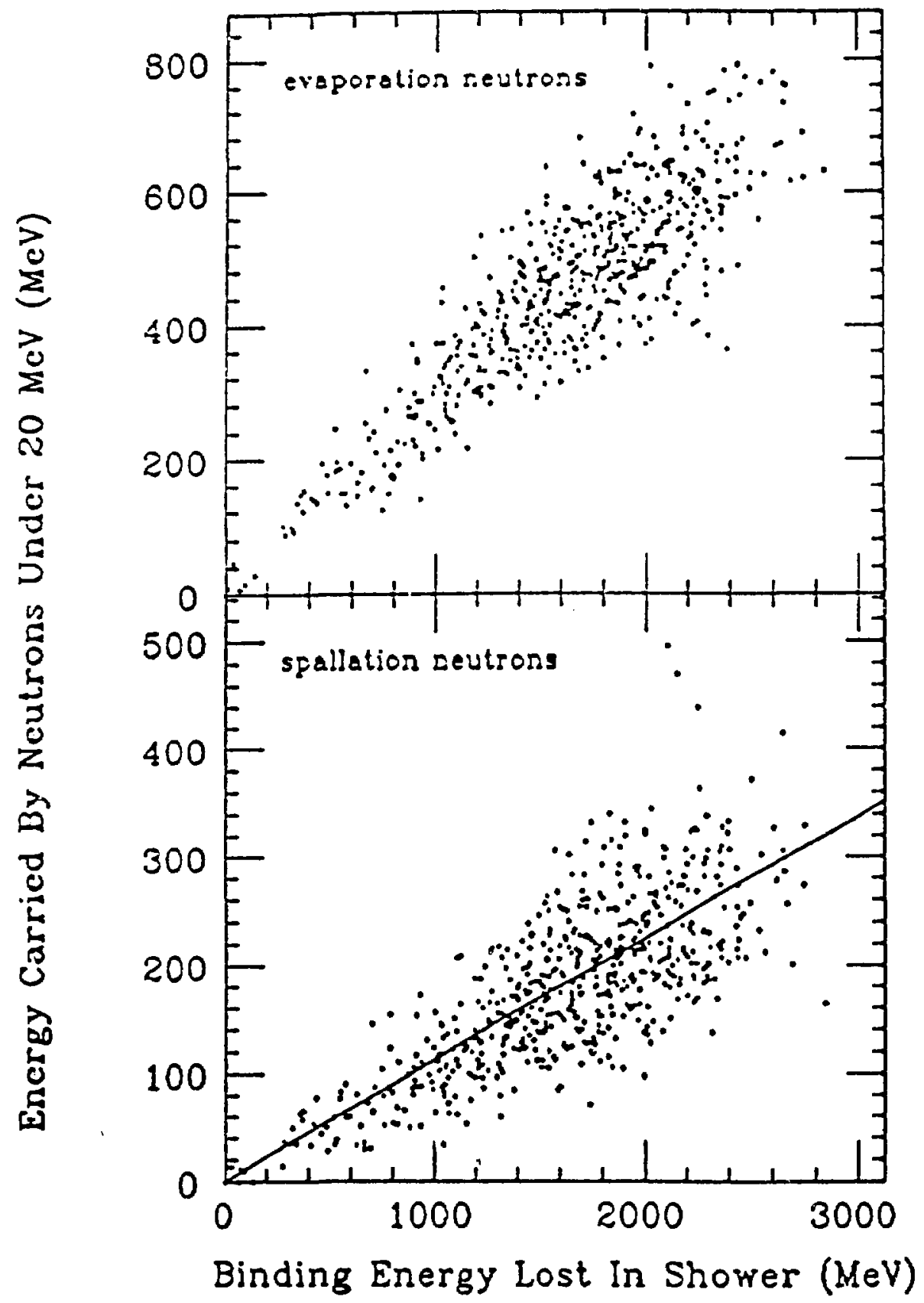

Fig. 3. The correlation of energy carried by neutrons below $20 \mathrm{MeV}$ to the binding energy lost in showers from $5 \mathrm{GeV} \pi^{-}$on a uranium-scintillator calorimeter made of 3 $\mathrm{mm}$ uranium plates and $3 \mathrm{~mm}$ scintillator sheets as calculated by HETC. The distribution is presented both for the evaporation neutrons and the spallation neutrons. The solid line shows the average trend of the data and is plotted for comparison with Fig. 4. 


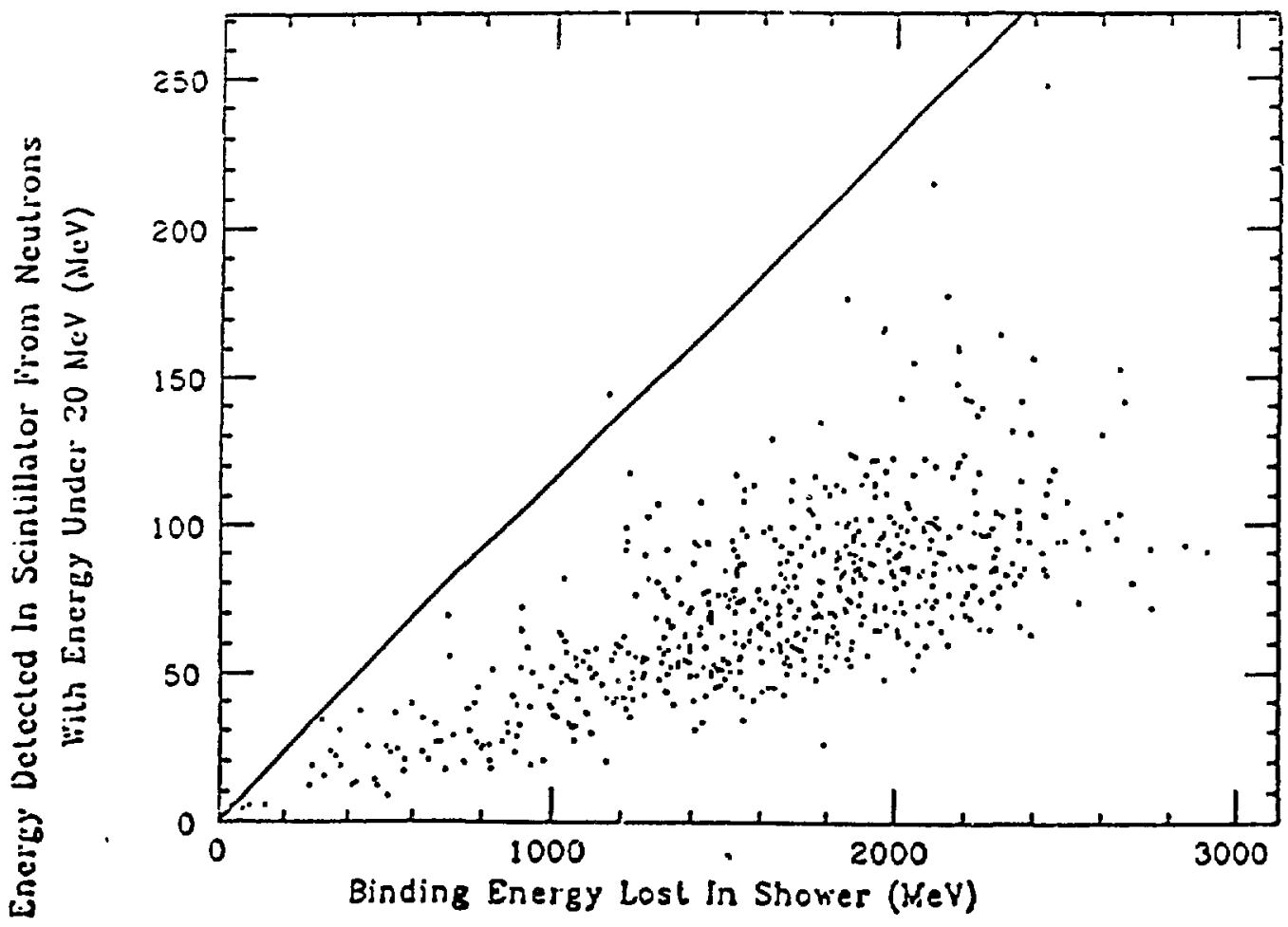

Fig. 4. Calculations of the energy detected in a $3 \mathrm{~mm}$ uranium $/ 3 \mathrm{~mm}$ scintillator calorimeter stack from the neutrons with energy below $20 \mathrm{MeV}$ versus the binding energy lost in showers from $5 \mathrm{GeV} \pi^{-}$. The solid line shows the average trend of the data in Fig. 3. See text for discussion. 
the hydrogen. Which will be discussed later. For comparison. the fraction of electromagnetic energy observed is about $6 \%$.

Since the transfer is so complete in the hydrogenous plastic scintillator. the relative contribution of the total signal can be varied by changing the relative thickness of the sampling layer and the uranium radiator, as was pointed out first in Ref. 7. This is illustrated in our calculations shown in Fig. 5. A calorimeter composed of three millimeter uranium plates and $3 \mathrm{~mm}$ plastic scintillator plates gives approximately the correct fraction of neutron coupling to bring the $\mathrm{e} / \mathrm{h}$ ratio to 1.0 . When the radiator thickness is increased to $6 \mathrm{~mm}$, the scintillator thickness must be considerably greater than $6 \mathrm{~mm}$ to achieve e/h $=1.0$. The neutron energy has been significantly reduced by the first $3 \mathrm{~mm}$ of scintillator and therefore the doubling of thickness does not double the neutron coupled energy.

To understand the difference between various hydrogenous and nonhydrogenous media consider the following concerning plastic scintillators and argon. First, the neutron cross sections in hydrogen continue to rise below $1 \mathrm{MeV}$, while the cross sections in argon are falling. This in itself means that a larger fraction of the soft neutron energy will be deposited in the plastic scintillat or than in the liquid argon. Secondly, the kinematic constraint on the energy transferred in a neutron-nucleus collision highly favors the transfer of energy to a lighter nucleus. The maximum possible recoil energy $\left(\left.E_{R}\right|_{\text {max }}\right)$ transferred from an incoming nonrelativistic neutron of kinetic energy $E_{n}$ to a recoil nucleus of atomic weight $A$ can be shown ${ }^{8]}$ simply from conservation of momentum and energy to be

$$
\left.E_{R}\right|_{\max }=\frac{4 . A}{(1+A)^{2}} E_{n}
$$

This means that in neutron-hydrogen collisions, the full energy of the neutron can be transferred, while in neutron-argon collisions the maximum energy which can be transferred is ten percent of the neutron energy.

Thirdly, the energy coupling is even more suppressed in liquid argon due to the saturation which occurs in regions of dense energy loss. In scintillators this phenomenon has been studied for many years and was modeled by Birks ${ }^{93}$ in a form that has come to be known as Birks' Law:

$$
\frac{d L}{d x}=\frac{S \frac{d E}{d x}}{1+k B \frac{d E}{d x}},
$$

where $\frac{d L}{d x}$ is the light yield per unit path length, $\frac{d E}{d x}$ is the specific energy loss for the charged particle, $S$ is the normal scintillation efficiency, and the $k B$ parameter describes the quenching which occurs for high density ionization. Typical $k B$ factors for scintillators are $0.01-0.02 \mathrm{gm} \mathrm{cm}^{-2} \mathrm{MeV}^{-1}$. Following the suggestion of Ref. 23, it has become customary to express the columnar recombination ${ }^{10}$, winch results in saturation in liquid argon ionization sampling calorimeters, also in the form of the Birks' Law. In this form the equivalent $k B$ factor for liquid argon is about $0.005 \mathrm{gm} \mathrm{cm}^{-2} \mathrm{MeV}^{-1}$, which is less saturating than scintillator. While the intrinsic saturation in liquid argon is less than 
$E / \pi$ vs. thickness $(U / \operatorname{scin})$

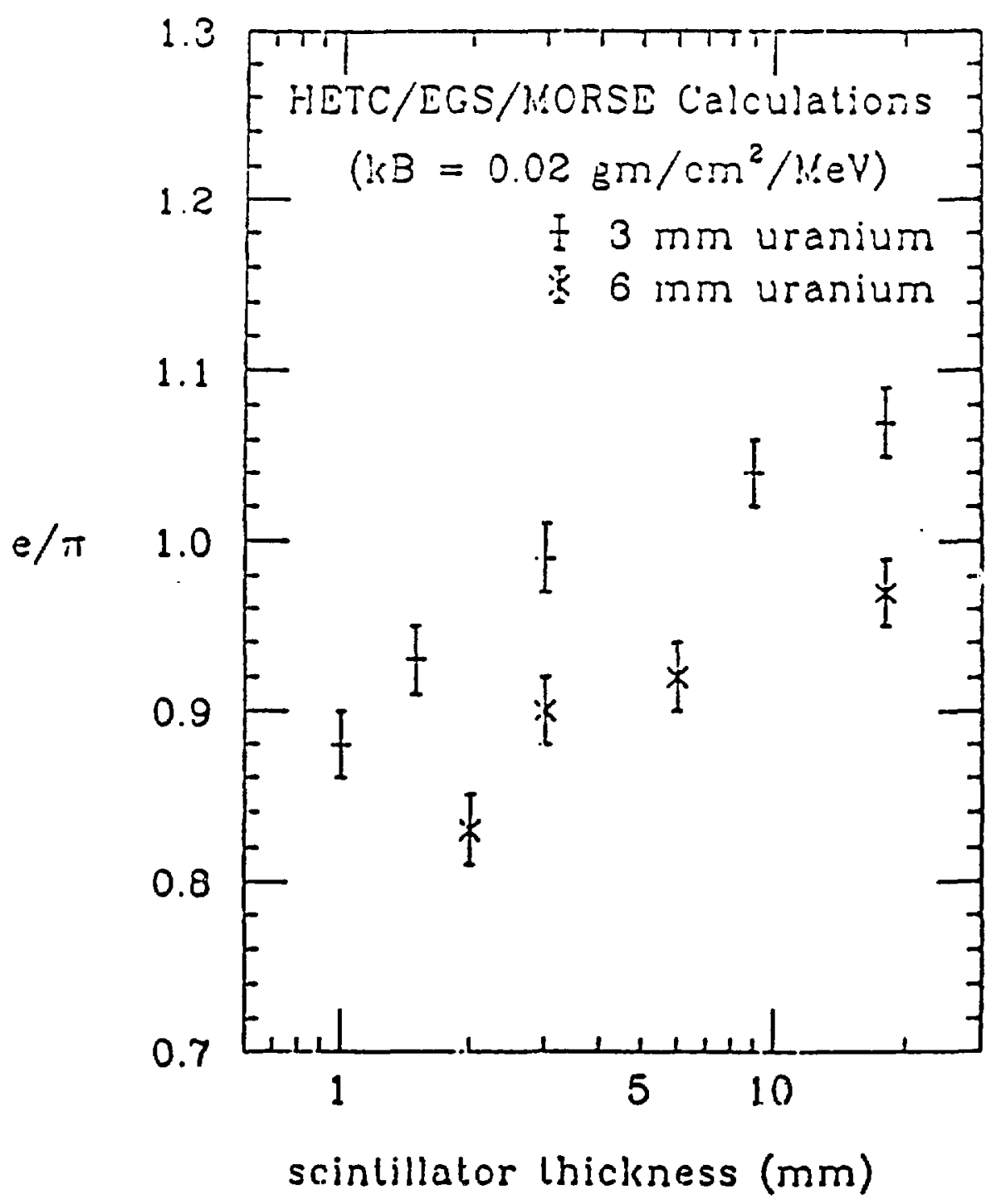

Fig. 5. The variation of $e / \pi$ with scintillator thickness for a set of uranium-scintillator calorimeter calculations for incident $5 \mathrm{GeV} \pi^{-}$. This figure illustrates the "tuning" possible with scintillate ', first noted in Ref. 7. 
in scintillator. the kinematic constraint on the energy transfers in neutronnucleus collisions leads to a greater suppression of the transfer of neutron encrgy to observable signal. In liquid argon, the highly ionizing recoil nuclei receive only one-tenth the relative energy of the less highly ionizing recoil protons in scintillator. For all practical purposes in high energy liquid argon calorimetry, the signal due to low energy $(<20 \mathrm{MeV})$ neutrons (except for the signal from produced gamma rays) is lost. ${ }^{11]}$ Silicon, in contrast to liquid argon and even scintillator material, shows very little saturation effect.

\subsection{Electromagnetic Sampling}

It is important to recognize the significant effects of reduced electromagnetic response in calorimeters composed of high $\mathrm{Z}$ radiator structures. ${ }^{2]}$ These "electromagnetic sampling inefficiencies" have been explained as resulting from the preferential absorption of low energy photons $(<1 \mathrm{MeV})$ in the high $\mathrm{Z}$ material and the effective electron path length stretching in the inactive media resulting from enhanced multiple scatiering in the high $\mathrm{Z}$ material. ${ }^{3,4, \pi]}$ Figure 6 display's how the preferential absorption of the low energy photons in the high $Z$ material occurs. Here the photon mass attenuation coefficient multiplied by the fraction of primary photon energy loss for argon and for lead can he seen. ${ }^{12]}$ Clearly, in the high $Z$ material, the relative absorption at low energy, say $\sim 100 \mathrm{keV}$, is much more effective in stopping the photons. The role of multiple scattering has also been clearly demonstrated by examining the effect of turning off multiple scattering in the electron-gamma shower program EGS. ${ }^{13]}$ Such calculations for $1 \mathrm{GeV}$ electron showers in uranium liquid argon sampling calorimeters show an increase of $10 \%$ in the signal deposited in the liquid argon as the electron path length stretching disappears. ${ }^{3,4]}$ The magnitude of this "electromagnetic sampling inefficiency" is often measured by comparing the response of a calorimeter for incident electrons to that anticipated for minimum ionizing particles. The fictitious fully contained minimum ionizing particles can be measured by scaling up the response of the calorimeter to muons. Such measurements find the ratio of average electron pulse height to the pulse height for the minimum ionizing particle to be roughly 0.6 to 0.7 for calorimeters constructed with high $Z$ radiators. The magnitude of this effect led to the prediction "that a lead calorimeter may also give $E M / H A D \approx$ 1." This prediction has now become reality. A lead-scintillator compensating calorimeter has been built and tested with scintillator thicknesses of one-fourth the lead thicknesses, a ratio designed to give the optimal electron to hadron ratio. ${ }^{14]}$ The reported $\mathrm{e} / \mathrm{h}$ ratio for this device is $1.05 \pm 0.04$ for energies over $10 \mathrm{GeV}$. Caution should be exercised, however, since this analysis is based on events selected by energy deposition. This selection could emphasize the electromagnetic parts of the hadronic shower and yield a biased $\mathrm{e} / \mathrm{h}$ ratio.

The understanding of the underlying phenomenology of compensating calorimeters is on solid ground and calculations can be done reliably. The requirements of the future hadron colliders demand that wise use be made of this understanding. 


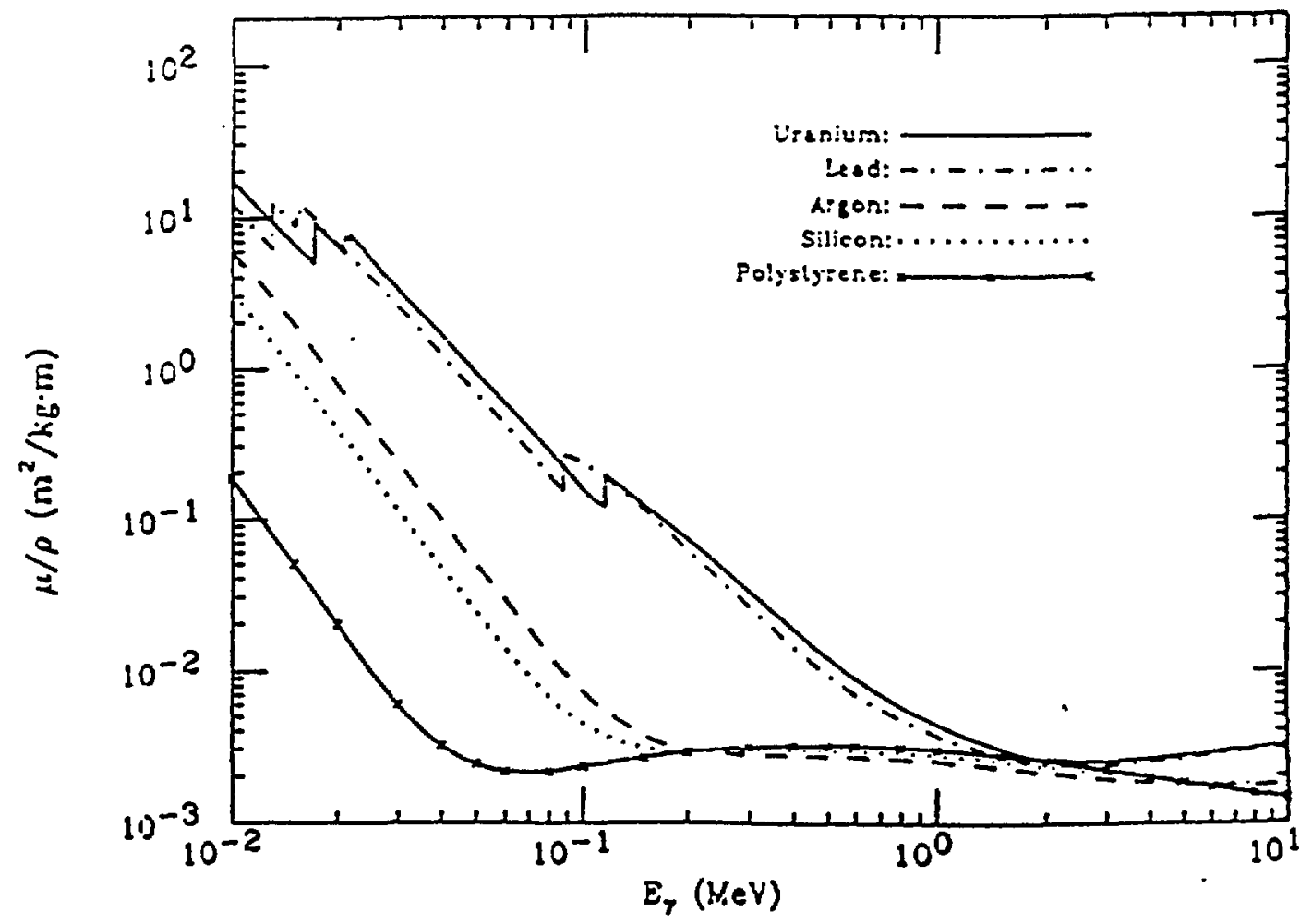

Fig. 6. Comparison of the photon mass attenuation coefficients multiplied by the fraction of primary photon energy loss for polystyrene, silicon, argon, lead, and uranium. 


\section{DETECTORS FOR THE SSC}

\subsection{Room Temperature Liquid Calorimetry}

Tetramethylsilane (TMS) and Tetramethylpentane (TMP) are the two liquids mentioned often concerning room temperature liquid calorimetry. Some of their advantages and disadvantages are listed in Table 1 . The two best advantages usually listed for these materials, at least in relationship to liquid argon, are first, the avoidance of cryogenic insulation which can lead to dead spaces in the detector system, and, secondly, good response to low-energy neutrons due to the high hydrogen concentrations. The response to low-energy neutrons will obviously depend on the saturation and recombination levels of TMS and TMP to low-energy recoiling protons. Some data are presently available which indicate that these levels are sufficiently low so that proton recoil detection is possible at a desirable level. Obviously, both the resolution and compensation characteristics of a warm liquid calorimeter will depend on this response. This dependence is illustrated in Figs. 7 and $8 .{ }^{15,16]}$

Since these design calculations and prototype studies, as illustrated by the data in Figs. 7 and 8, experimental results have been obtained on a UA1 uranium-TMP calorimeter module. The device is composed of two parts. The front end contains a fine sampling electromagnetic calorimeter followed by a courser sampling hadronic (not completely containing) calorimeter composed of uranium and TMP. Behind this module was placed an Fe/scintillator catcher calorimeter to complete containment of the hadronic cascade. Considering the limited amount of uranium in this calorinetry, good energy resolution and compensation has been obtained. Analytic fits to the electron and hadron energy resolution yields $13.3 \% / \sqrt{E}+0.5 \%$ and $47.5 \% / \sqrt{E}+7 \%$, respectively. The compensation as a function of energy is given in Fig. 9. For comparison, $\mathrm{U} /$ scintillator and $\mathrm{Fe} /$ scintillator calorimeter $\mathrm{e} / \pi$ ratios are also presented.

The main radiation damage to TMS or TMP is decomposition into other products. For dose levels on the order of $10^{5} \mathrm{Gys}$, approximately $1 \%$ of the liquid will decompose. ${ }^{18]}$ These dose levels per year can be achieved in SSC detectors for pseudorapidity levels greater than 3. This decomposition can lead to gas pressure buildup, decrease in electron lifetime, reaction of products with container vessel, etc. All of these problems must be considered and methods chosen to overcome them.

\subsection{Silicon Calorimetry}

The use of silicon as the active media for an SSC detector has been suggested rather recently, and in this short timeframe, substantial R\&D has been carried out. Some of the advantages and disadvantages of silicon are listed in Table 2 and a few of the more important issues are discussed below.

The fundamental question that needs to be addressed is what will be the $\mathrm{e} / \mathrm{h}$ ratio for silicon-based calorimeters. One property of silicon that plays an important role in this study is the extreme linearity of silicon up to very large stopping power. ${ }^{19]}$ That is, silicon exhibits very little saturation. It has been demonstrated that saturation prevents full compensation in currently designed uranium-liquid argon calorimeters, so this could be a very important factor in silicon calorimeters. Naively, it is expected that very good results can be obtained for silicon calorimeters. 


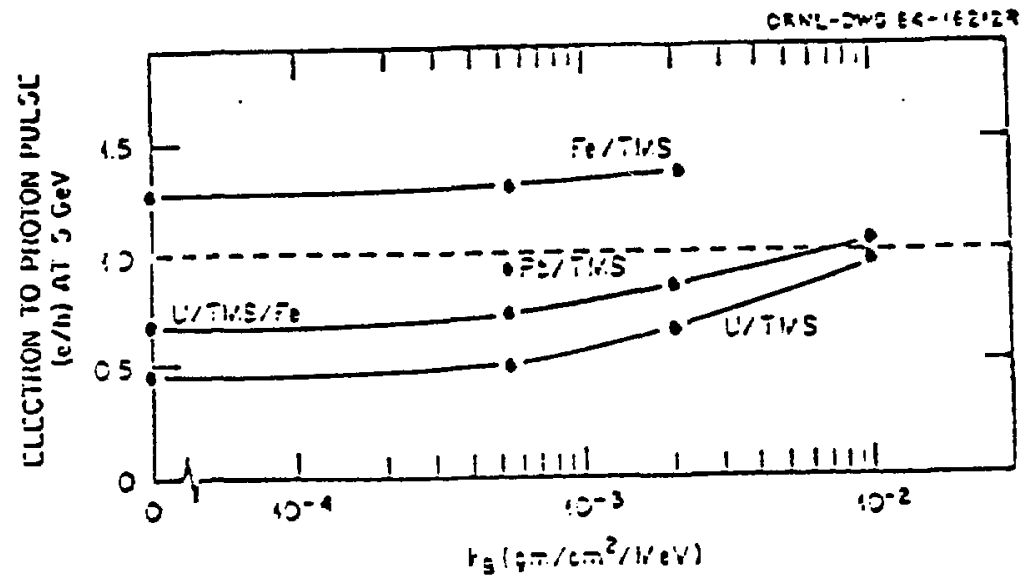

Fig. 7. The $e / \pi$ ratio is strongly dependent on the saturation and recombination levels in tetramethylsilane (TMS) or tetramethylpentane (TMP).
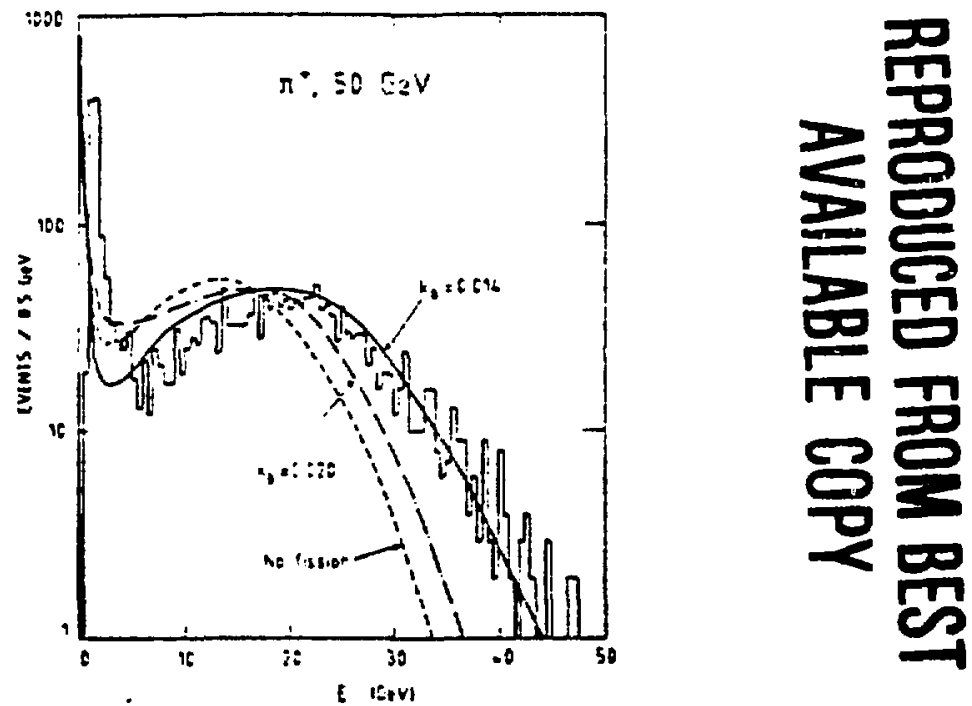

Fig. 8. The resolution of TMS- and TMP-based calorimeters is strongly dependent on the saturation and recombination levels of these materials.

The linearity of response of silicon to large energy deposition densities is summarized in Fig. 10. This figure shows the relative response of a recoil ion of maximal energy in neutron scattering. ${ }^{20]}$ One sees that even for silicon ions as low as $100 \mathrm{keV}$ nearly one-half of the deposited energy is detected as observable output signal. Given these evidences of very limited saturation in silicon detectors a very good response to some components of the low energy development of hadronic showers can be expected. It should be remembered that part of the signal due to stopping power will not be detected, that is, the Coulomb scattering part. The Linclhard et al. theory, as indicated in the figure represents the fraction that is due to electron ionization and excitation, and any 


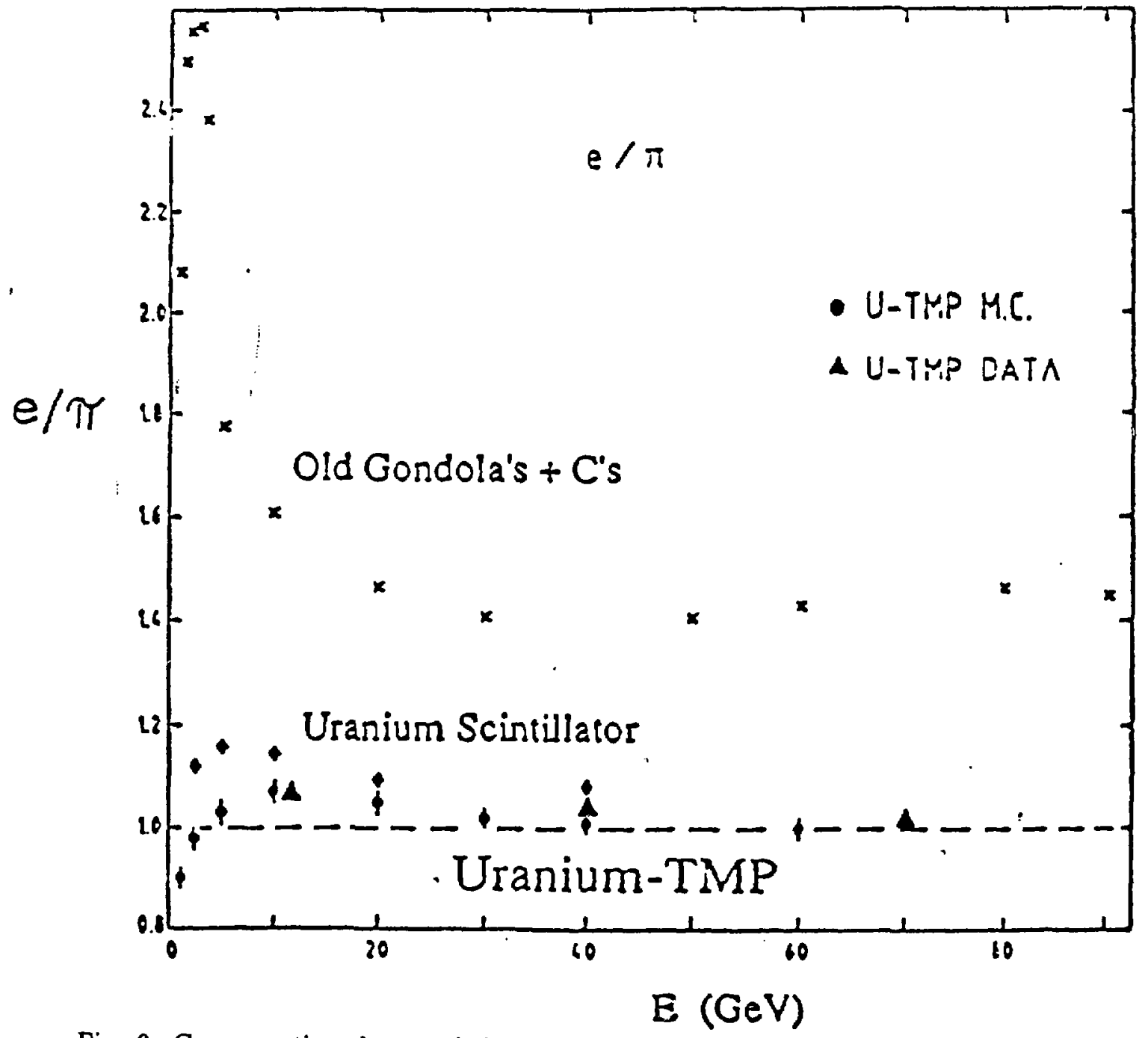

Fig. 9. Compensation characteristics of a U/TMP/Fe/scintillator calorimeter. Because of the small amount of $U$, the compensation is strongly controlled by electromagnetic
sampling inefficiencies. 
experimental data deviation from this curve should represent the amount of true sa:uration and/or recombination.

Table 1. Advantages and Disadvantages of Room Temperature Liquid Calorimetery Tetramethylsilane (TMS) and Tetramethylpentane (TMP) Calorimetry

- Advantages

- Good absolute stable signal (easily calibrated)

- Spatially uniform in charge collection

- Easy to segment

- Requires little space for readout electronics

- Good response to low energy neutrons (?)

- Noncryogenic (no dead spaces needed for insulation)

- Disadvantages

- Signals are generally small (field dependent but TMP a factor of 3 less than Ar; however, TMS $70 \%$ of Ar)

- Very sensitive to impurities (ppm level)

- New technology (UA1 at CERN: TMP; Engler et al., at KfT: TMS)

- Low boiling point $\left(112 .^{\circ} \mathrm{C}, \mathrm{TMP}, 26.5^{\circ} \mathrm{C}\right.$, TMS) and flash point (TMP $7^{\circ} \mathrm{C}, \mathrm{TMS}$ $-18^{\circ} \mathrm{C}$ )

- Radiation damage (?)

- Slow charge collection (50 ns for $1 \mathrm{~mm}$ TMS gap at $20 \mathrm{kV} / \mathrm{CM}, 150 \mathrm{~ns}$ for TMP)

- Compensation Characteristics

- Depends on level of saturation and recombination

- Energy Resolution

- Depends on level of saturation and recombination 
Table 2

Advantages and Disadvantages of Silicon Calorimetry

\section{Silicon Calorimetry}

- Advantages

- Good absolute stcble signal (easily calibrated)

- Spatially uniform in charge collection

- Easy to segment

- Requires little space for readout electronics

- Nonsaturating readout (well, almost)

- Fast charge collection

- Disadvantages

- Radiation damage (sensitive to low energy neutrons)

- Small sampling fraction ( $0.4 \mathrm{~mm}$ thick)

- Low sensitivity to $\mathrm{MeV}$ energy neutrons

- Cost (cost $/ \mathrm{cm}^{2}$ of active materiai $\sim 2-3$ times too high)

- New technology

- "Texas tower events"

- Compensation Characteristics

- Probably can be made compensating

\section{- Energy Resolution}

- Good, but not excellent 


\section{REPRODUCED FROM BEST AVAILABLE COPY}

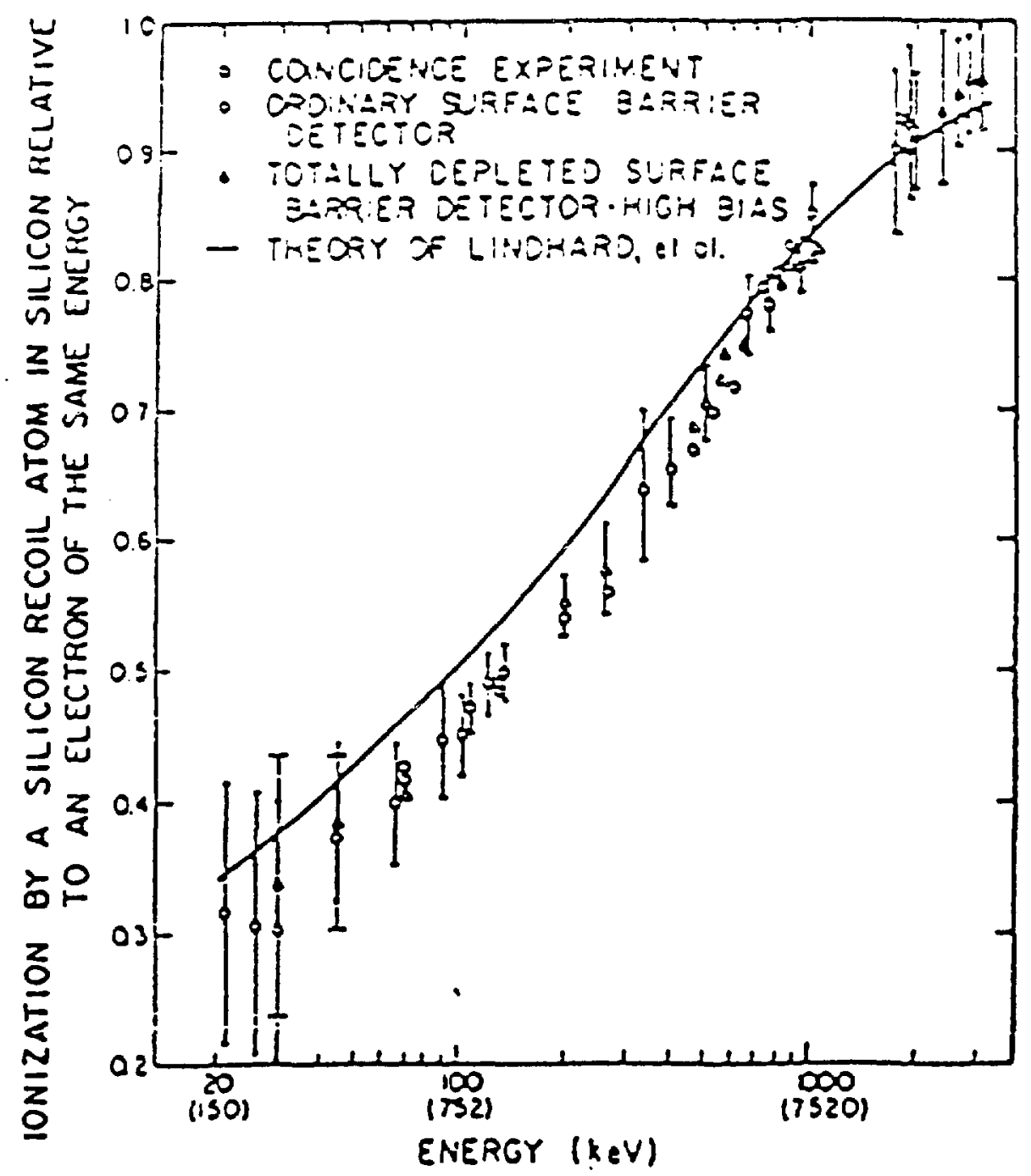

Fig. 10. Pulse height produced by a $\mathrm{Si}$ recoil atom relative to that of an electron of the same energy in $\mathrm{Si}$ as a function of $\mathrm{Si}$ recoil energy. The incident monoenergetic neutron energy necessary to produce the denoted recoil energy is shown in parenthesis. (From Ref. 20) 
Figure 11 shows the calculated response distribution for $10 \mathrm{GeV} \pi^{-}$incident on a silicon calorimeter with $5 \mathrm{~mm}$ thich uranium radiators and $400 \mu \mathrm{m}$ fully depleted silicon detertors sandwich of between two layers of $5 \mathrm{~mm}$ thick G10. Therefore, the total layer thickness is $15.4 \mathrm{~mm}$ and the depth of the stack extends for 150 readout and radiator layers. The transverse dimensions of the stack have been taken to be $100 \times 100 \mathrm{~cm}^{2}$. The charge collection is cut off after 50 nanoseconds. The resulting energy resolution $(\sigma / E)$ is $21.5 \%$ and the $\mathrm{e} / \mathrm{h}$ ratio of is 1.07 , is close to compensation. ${ }^{21]}$ In fact, the two layers of $\mathrm{G} 10$ in this stack are increasing the value of $e / h$ as the neutron energy from the uranium is being deposited in the G10 through the large energy transfers via hydrogen. Additionally, significant transfers occur to the carbon and oxygen in the G10.

Figure 12 presents the event by event correlation of the energy detected in the silicon from the low energy $(<20 \mathrm{MeV}$ ) neutrons to the binding energy lost in the hadronic cascade. While the correlation is strong, this figure shows much larger fluctuations in detected energy than was seen for a uranium-scintillator calorimeter. ${ }^{22}$ This fluctuation depends on the number of neutrons actually detected. Given the very thin detection layers, the number of neutrons actually detected is small. Unlike the normal shower fluctuations which vary as $\sqrt{t_{c \epsilon l l}}$ and are dominated by the thickness of the radiator, the fluctuation on neuiron response is dominated by the thickness of the silicon $\left(t_{S_{i}}\right)$, and scales as $\sqrt{t_{S_{i}}}$. Therefore, the sampling fluctuations for the charged hadrons in the hadron cascades will be similar to any other readout medium, but the additional larger fluctuations on the neutrons will add to the width of the energy distribution, preventing excellent low energy resolution even for perfectly compensating calorimeters.

Figure 13 presents a series of calculated results for calorimeters constructed with $2 \mathrm{~mm}$ uranium radiators, followed by a layer of $\mathrm{G} 10$ of varying thickness and a $400 \mu \mathrm{m}$ fully depleted silicon detector. As before, $k B=0$, the time cut is 50 nanoseconds, and the transverse size is taken to be $100 \times 100 \mathrm{~cm}^{2}$. The stacks contain 330 layers of uranium, G10, and silicon. It can be seen that with no G10 the condition of overcompensation is obtained due to the sensitivity of the silicon detectors to very low energy particles. As the G10 is added, the $\mathrm{e} / \mathrm{h}$ ratio increases due to the removal of neutron energy by the G10, meaning less energy is available to be deposited in the silicon. Naively, one might expect that the neutrons interacting in the G10 would contribute to the response of the silicon by knocking protons into the detectors. It has been proposed $^{23]}$ that coupling a hydrogenous material to liquid argon, for example, would be a possible method for restoring its potential for compensation.

However, the efficiency and range of the protons produced in the G10 is not large enough to have much of an effect on the total response of a detector of 400 $\mu \mathrm{m}$ thichness. ${ }^{24]}$ It can also be seen in Fig. 13 that the energy resolution reaches a minimum at approximately the point where the $\mathrm{e} / \mathrm{h}$ ratio passes through one, as would be expected for a compensating calorimeter. The value of this resolution is not as small as the values achieved in scintillator because of the larger sampling fluctuations involved here, as described previously.

Several cases of silicon calorimeters have also been studied with different radiator materials, specifically one employing lead and one employing iron. For 


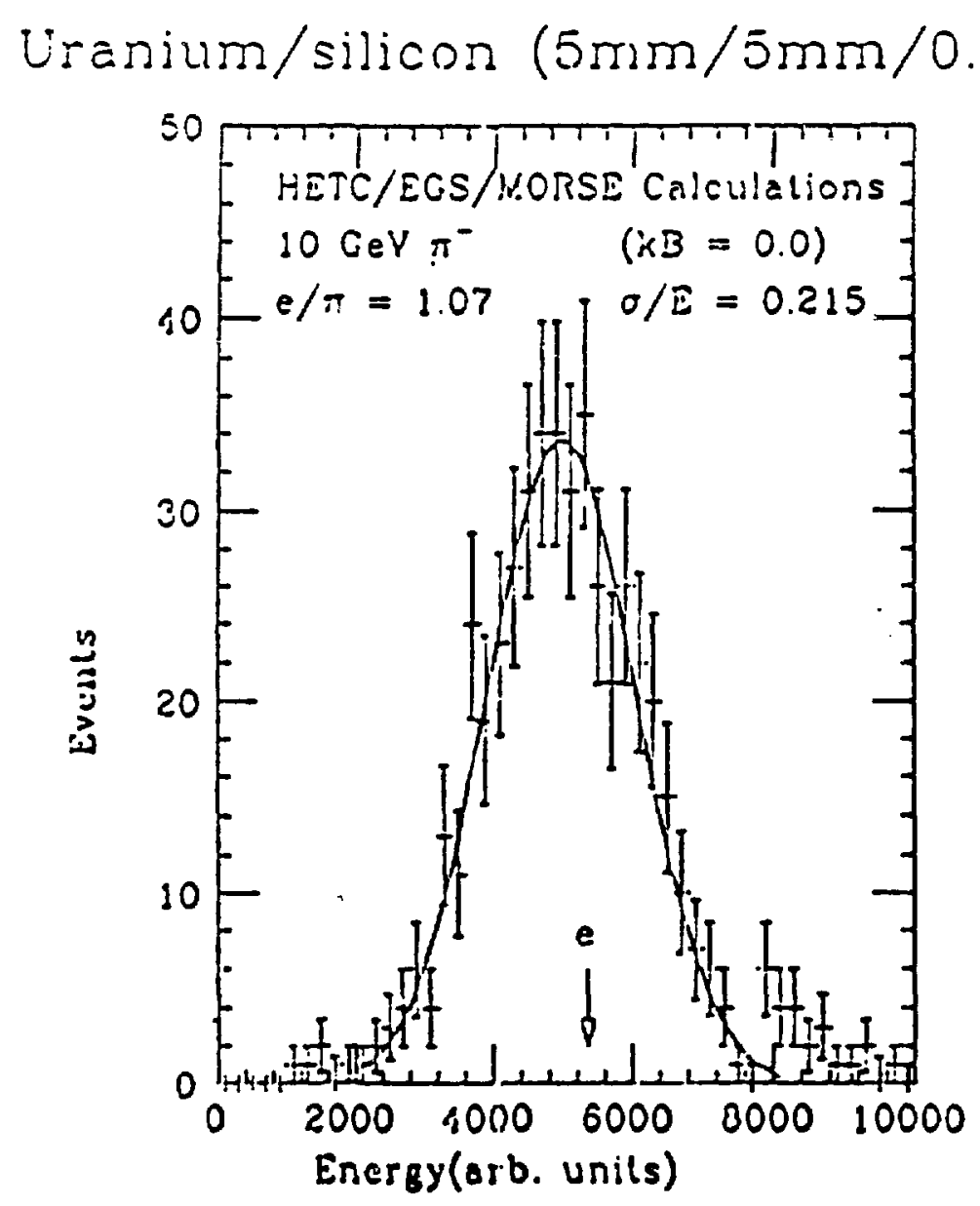

Fig. 11. The calculation of the measured hadron energy distribution for $10 \mathrm{GeV} \pi^{-}$ incident on a uranium-silicon calorimeter with $5 \mathrm{~mm}$ thick uranium radiators and $400 \mu \mathrm{m}$ fully depleted detectors imbedded in $5 \mathrm{~mm}$ thick G10 supports. 


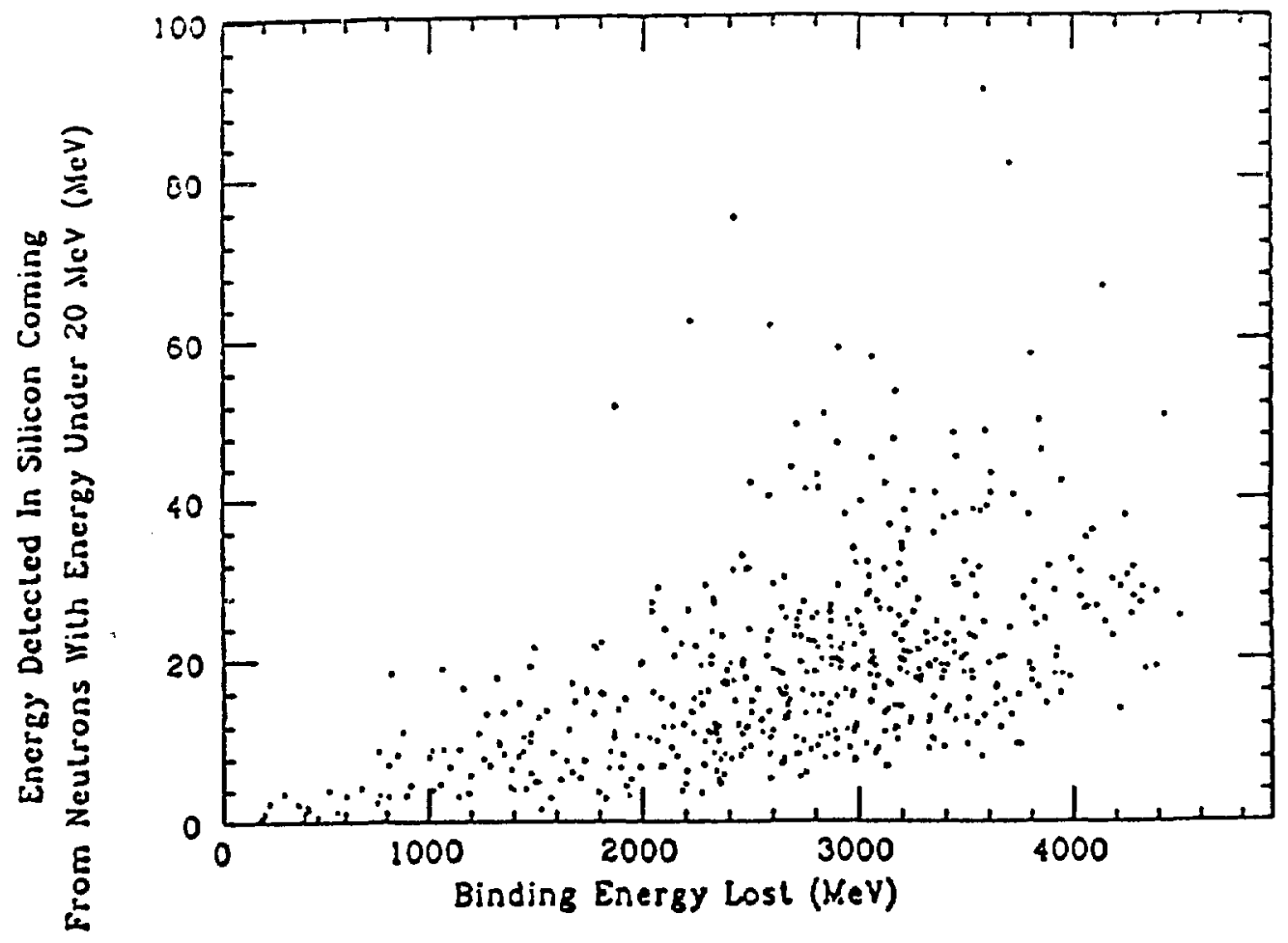

Fig. 12. The correlation of detected low energy $(\mathrm{E}<20 \mathrm{MeV})$ neutron energy in the uranium-silicon calorimeter with the binding energy lost in the hadronic cascade. 


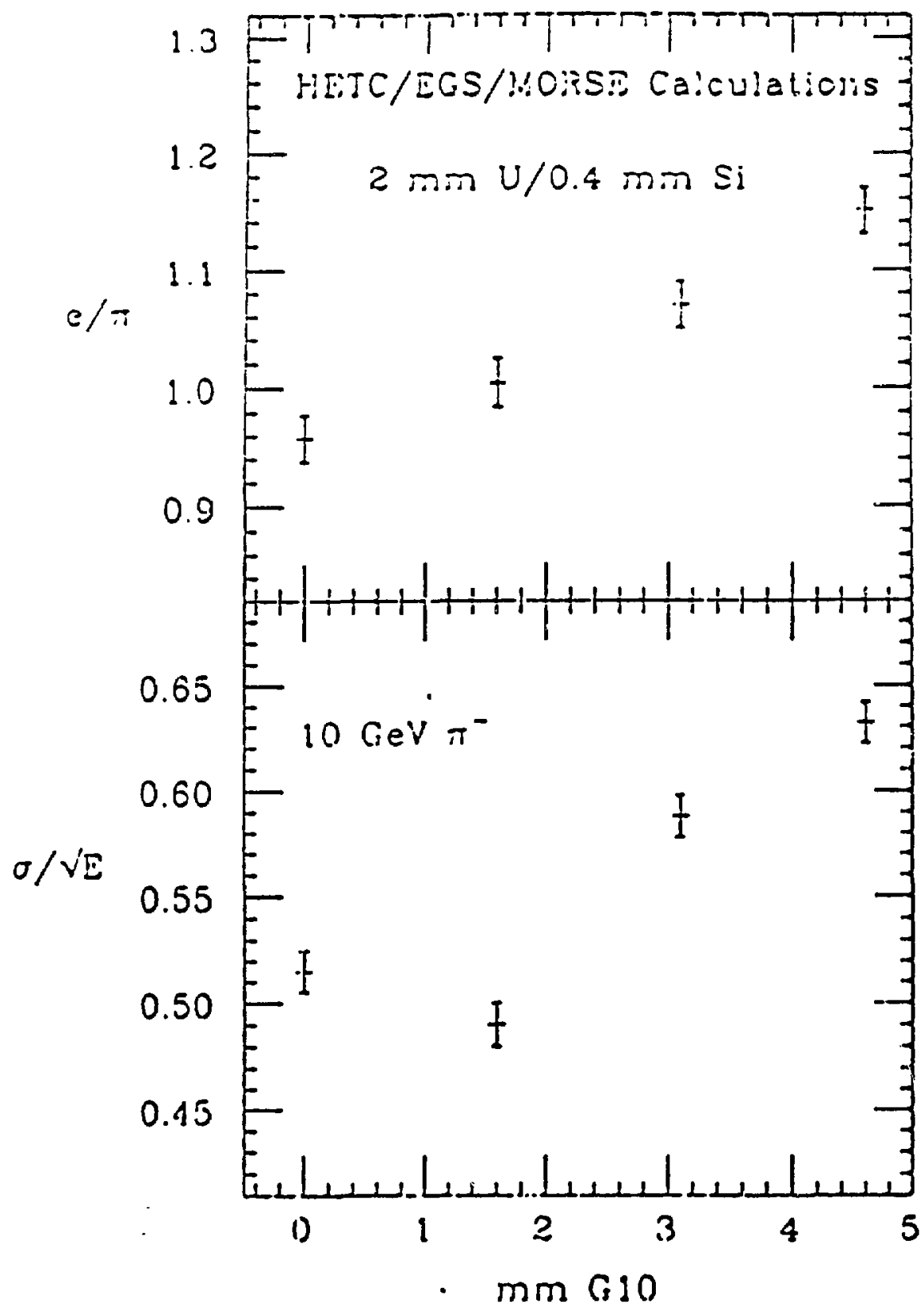

Fig. 13. The calculated variation of $\mathrm{e} / \pi$ and $\sigma / \sqrt{E}$ with the thickness of a G10 sheet placed between the $2 \mathrm{~mm}$ uranium plates and the silicon detectors in a uranium-silicon calorimeter for incident $10 \mathrm{GeV} \pi^{-}$. Without $\mathrm{G} 10$, a slight overcompensation conditions exists. 
a lead calorimeter wirl $3 \mathrm{~mm}$ thich radiators and $400 \mu \mathrm{m}$ fully depleted silicon detector with no air gaps and the same transierse geometrical constraints and time cuts as before, the calculated results yielded an expected e/h ratio of 1.16 with an energy resolution of $\frac{\sigma}{\sqrt{E}}=47 \%$ for $10 \mathrm{GeV} \pi^{-}$. For an iron calorimeter with $7.5 \mathrm{~mm}$ radiators, followed by a $1.3 \mathrm{~mm}$ air gap, a $1 \mathrm{~mm}$ G10 layer, $400 \mu \mathrm{m}$ of fully depleted silicon, a $1 \mathrm{~mm} \mathrm{G10} \mathrm{layer,} \mathrm{and} \mathrm{another} 1.3 \mathrm{~mm}$ air gap, the e/h ratio increases to 1.29 . For the $10 \mathrm{GeV} \pi^{-}$the resolution is $\frac{\sigma}{\sqrt{E}}=62 \%$. Even for the iron, the role of low energy detection efficiency can be seen in the $e / h$ ratio, and for the lead, the $\mathrm{e} / \mathrm{h}$ ratio of 1.16 is within reach of the values $w$ hich have been touted as acceptable for future "compensating" calorimeters. ${ }^{25}$ ]

In connection with these calculations, the attractiveness of a low- $Z$ radiator such as iron should be pointed out. Iron has several advantages compared to uranium, for example. In iron, less binding energy loss in inelastic collisions is realized, and this factor therefore contributes less to the energy spread than in calorimeters employing higher $Z$ radiators. Secondly, connected with this is the fact that fewer neutrons are produced in these collisions and the produced neutrons are a source of radiation damage at high luminosity colliders. Thirdly, uranium is more expensive than iron. ${ }^{26}$ ] Finally, uranium is difficult to obiain and to work with. In spite of these advantages for low $\mathrm{Z}$ over high $\mathrm{Z}$, it is difficult to anticipate a practical design of a compensating iron calorimeter. As we have seen, the electromagnetic sampling inefficiencies are an important factor in achieving compensation. These effects are small in iron due to the small value of the charge of the iron nucleus. As a result, the response of an iron calorimeter to electromagnetic and non-electromagnetic energy deposition is much different, yielding large fluctuations in the pulse height distribution. The small binding energy losses are overcome by the fluctuations between electromagnetic and nonelectromagnetic energy. It is conceivable to imagine a combination of low$Z$ and high- $Z$ material which will combine the advantages of each material and approach a resolution determined by the small binding energy losses of iron.

Radiation damage to $\mathrm{Si}$ detectors is still the largest drawback to the utilization of $\mathrm{Si}$ in SSC calorimeters. For pseudorapidity levels greater than 2 to 3 , neutron fluences on the order of $10^{13}$ to $10^{14} / \mathrm{cm}^{2} / \mathrm{yr}$ can be expected and damage from lattice displacement can be anticipated. Additional R\&D to develop rad hard $\mathrm{Si}$ is needed and some recent experiments appear encouraging.

\subsection{Liquid Argon Calorimetry}

Liquid argon calorimetry is very established as a potential candidate for utilization in one of the first SSC detectors. Table 3 lists some of the advantages and disadvantages of liquid argon calorimetry.

Even though liquid argon calorimetry represents an established technology, there are several disadvantages which limit its usefulness. The first of these is that liquid argon requires a cryogenic system and therefore large dead spaces are needed for insulation. A large amount of R\&D is currently underway (see these Proceedings) to minimize the effect of the dead space.

The other major problem associated with liquid argon is its nonsensitivity to low-energy neutrons. Because of the nondetectability of the energy contained in the low-energy neutrons, a fine sampling uranium liquid argon calorimeter can not totally compensate. This noncompensation is illustrated in Fig. 14. ${ }^{27}$. As 
the calorimeter sampling is increased, the $e / \pi$ ratio moves away from unity. To decrease this ratio, the electromagnetic sampling inefficiency must be used. This requires increasing the uranium thickness. However, increasing the uranium thickness will increase the resolution. This is illustrated in Fig. 15. $\left.{ }^{27}\right]$

Radiation damage to liquid argon should not be a problem unless methane or other substances are added. Then, similar problems as with warm liquid calorimetry can be expected, i.e., pressure due to $\mathrm{H}_{\text {: }}$ gas buildup, change in electron lifetime, and drift velocity, etc.

Table 3

\section{Advantages and Disadvantages of Liquid Argon Calorimetry}

\section{Liquid Argon Calorimetry}

\section{- Advantages}

- Good absolute stable signal (easily calibrated with sources)

- Spatially uniform in charge collection

- Easy to segment

- Requires little space for readout electronics

- Good sirong signals

- Established technology

- Nominal radiation damage

\section{- Disadvantages}

- Requires cryogenic system (large dead spaces needed for insulation)

- Very sensitive to impurities

- Poor response to low energy neutrons (energy transfer to Ar atoms small, satur: 'on and recombination levels high on low energy (MeV) recoiling $\mathrm{Ar}$ \& , oms)

- Slow charge collection

- Compensation Characteristics

- Relies on electromagnetic sampling inefficiency

\section{- Energy Resolution}

- Good (low energy neutrons not detectable) 


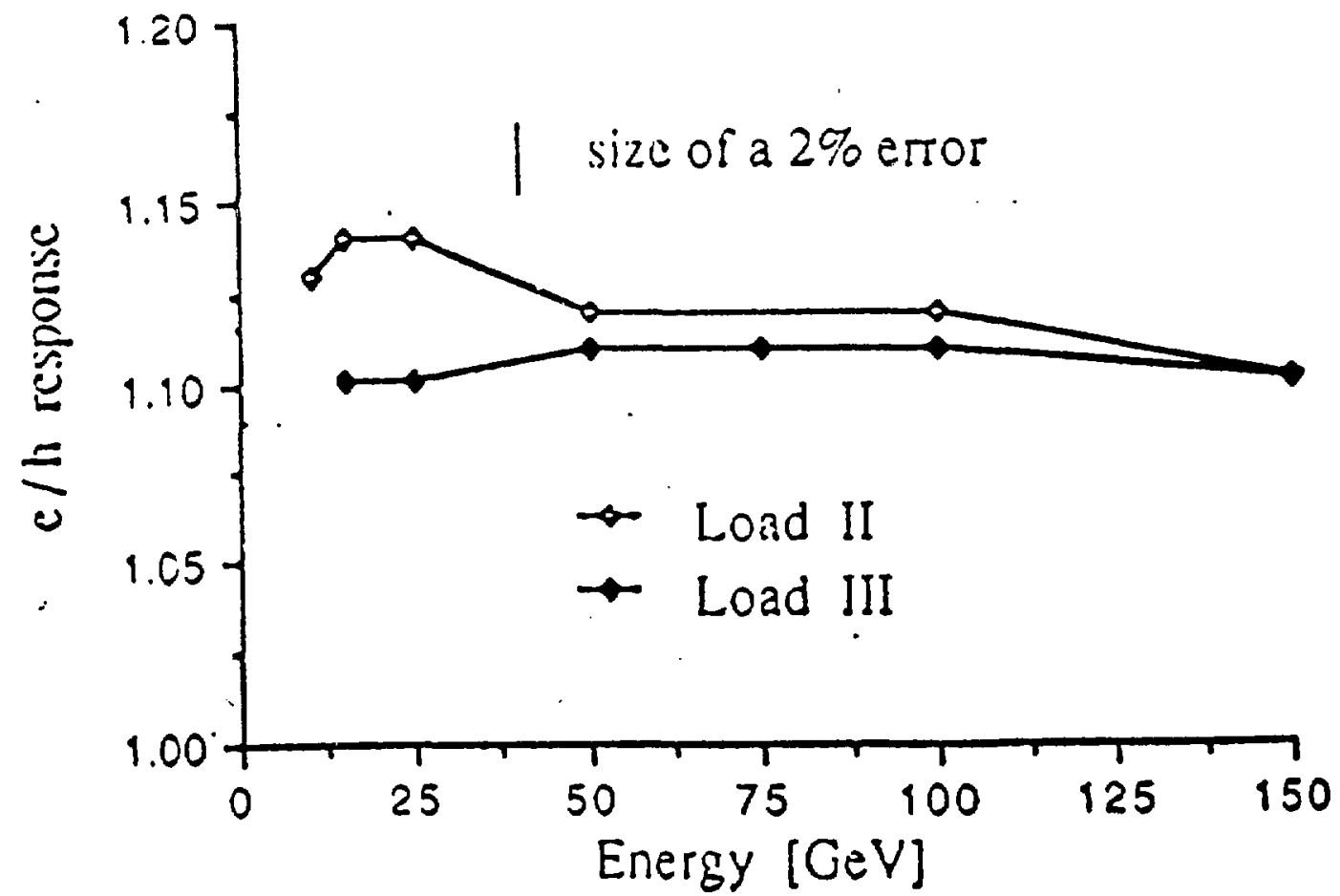

The ratio of electron over hadron response $(e / h)$ in configurations, Load II and Load III.

Load II $30-2 \mathrm{~mm}$ U plates Load III $16-4 \mathrm{~mm}$ U plates $112-4 \mathrm{~mm}$ U plates $\quad 56-8 \mathrm{~mm}$ U plates 24-19 $\mathrm{mm}$ Cu plates 24-19 $\mathrm{mm}$ Cu plates

$1.6 \mathrm{~mm}$ Ar-1.6 mm signal board-1.6 mm Ar

Fig. 14. The e/ $\pi$ ratio for two different uranium-liquid argon calorimeters. 


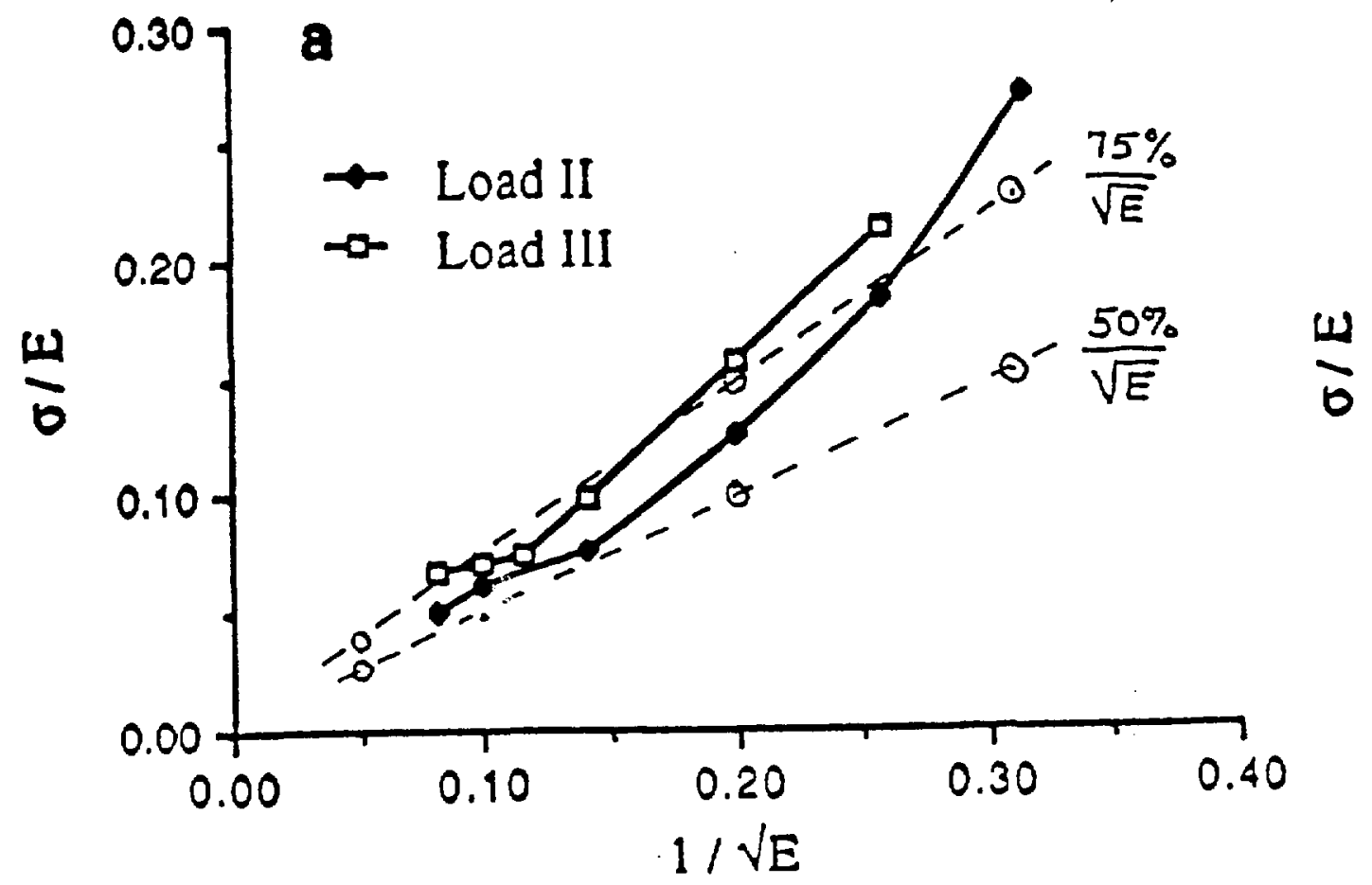

Fig. 15. Energy resolution for hadrons in Load II and III without any cuts.

\subsection{Scintillator Calorimetry}

Scintillator calorimetry enjoys the benefits of long-term $R \& D$. The very first true calorimeters were composed of scintillators and some other inactive material such as iron. The advantages and disadvantages are given in Table 4. Other than being an established technology, the other extremely important advantage of scintillator calorimetry includes good sensitivity to low energy neutrons. This fact alone allows for compensation (when included with a high $\mathrm{Z}$ passive material) and good energy resolution; in fact, the currently best obtained: $\sim 35 \% / \sqrt{E}$.

The level of saturation that can be expected in scintillators is illustrated in Fig. 16. Even though some saturation exists, low energy protons resulting from neutron collisions are detectable. The total energy deposited into the scintillator material located in a uranium calorimeter is dominated by the energy contained in the low energy neutrons $\left(E_{N} \leq 20 \mathrm{MeV}\right)$. This is illustrated in Fig. 17. Even when saturation effects are included, these low energy neutrons still represent a large percentage of the total detectable energy.

The energy resolution and compensation of a uranium/copper scintillator calorimeter are given in Figs. 18 and 19 , respectively. ${ }^{28]} \mathrm{A}$ comparison with these data and calculated data ${ }^{23]}$ is given in Table 5. As can be seen, the agreement between the calculated and experimental data is quite good giving credibility to both sets of data.

Radiation damage will also be a problem for scintillator calorimeters in the pseudorapidity range greater than 2.5 to 3 . Rad hardened plastics will have to be used to reduce the darkening of the material which will have a strong detrimental effect on the light collection. 
Table 4

Advantages and Disadvantages of Scintillator Calorimetry

\section{- Advantages}

- Fast light collection (25-50 nsec)

- Small nonsaturating readout (well, almost)

- Established technology

- Good sensitivity to low energy neutrons

- Best resolution obtainable $(\sim 35 \% / \sqrt{E})$

- Low in cost

- Disadvantages

- Radiation damage

- Nonuniform light collection

- Can not easily be highly segmented

Table 5

Summary of Calculated and Experimental Energy Resolutions and Compensations for U/Plastic and $\mathrm{Cu} /$ Plastic Calorimeters $\left(5 \cdot \mathrm{GeV} \pi^{-}, \mathrm{kB}=0.02 \mathrm{gm} / \mathrm{cm}^{2} / \mathrm{MeV}\right.$ )

\begin{tabular}{lccccc} 
& \multicolumn{2}{c}{$\sigma / \sqrt{E}$} & \multicolumn{2}{c}{$\mathrm{e} / \pi$} \\
& $\underline{1}$ & Data & Calculated & Data & Calculated \\
All-Uranium & 33 & 37 & 1.1 & 1.02 \\
50-50 Copper-Uranium & 38 & 39 & 1.2 & 1.12 \\
All Copper & 51 & 54 & 1.4 & 1.53
\end{tabular}

Segmentation is also a problem associated with scintillation calorimetry. However, several designs ${ }^{29}$ ] are being considered, which maintain good energy resolution and yet are highly segmented. Prototype studies for these detectors are being planned.

\subsection{Summary}

All of the presented detector systems have potential application as the calorimeter in the main SSC detector. Within certain limits, acceptable energy resolution and compensation can be expected. A summary of this type of data is given in Table 6. Each detector system has unique and common difficulties that must be overcome before any one of them can be seriously considered as the prime contender for the main SSC calorimeter. 


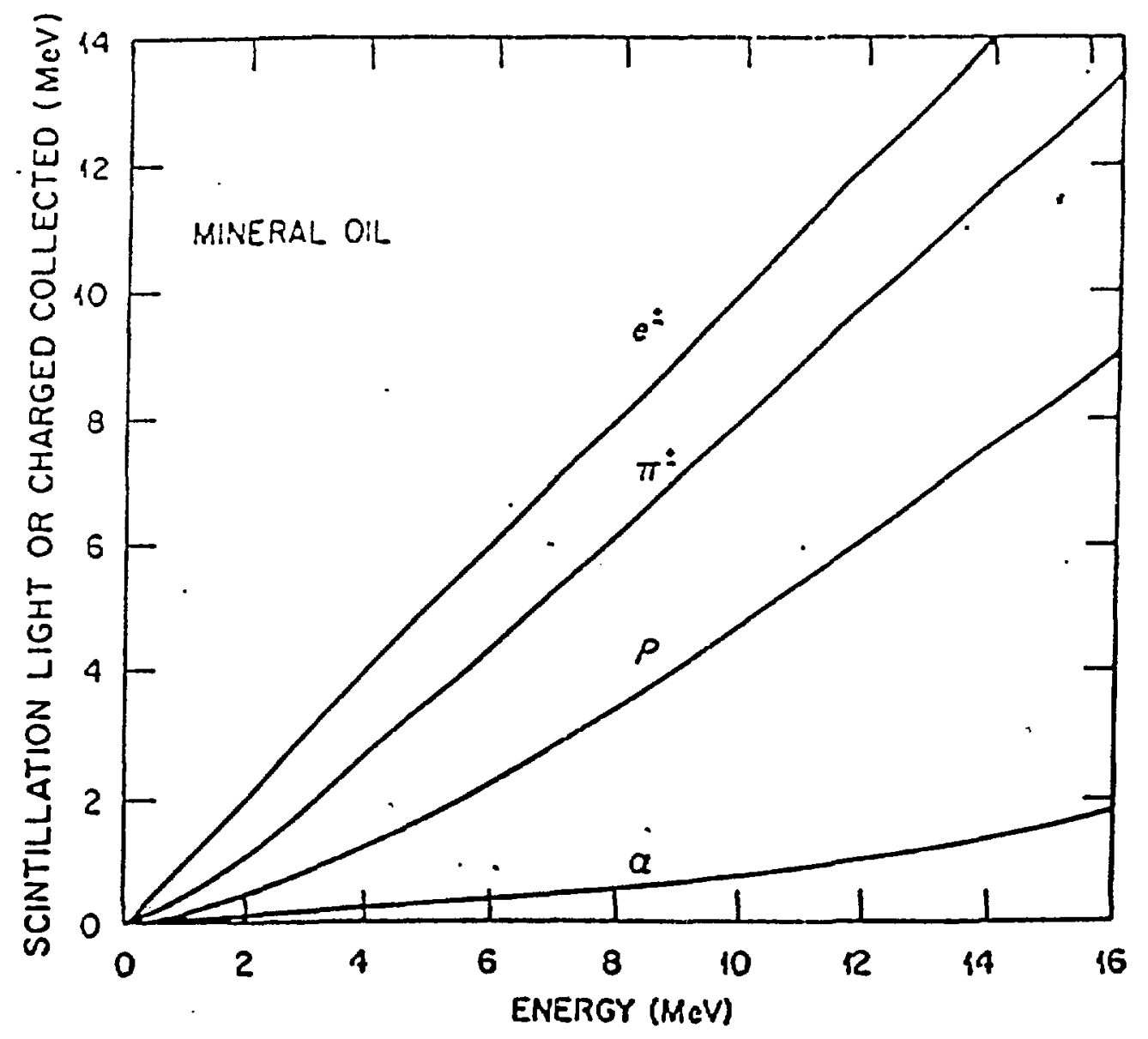

Fig. 16. Saturution levels in scintillating material for various particle types and as a function of energy. 


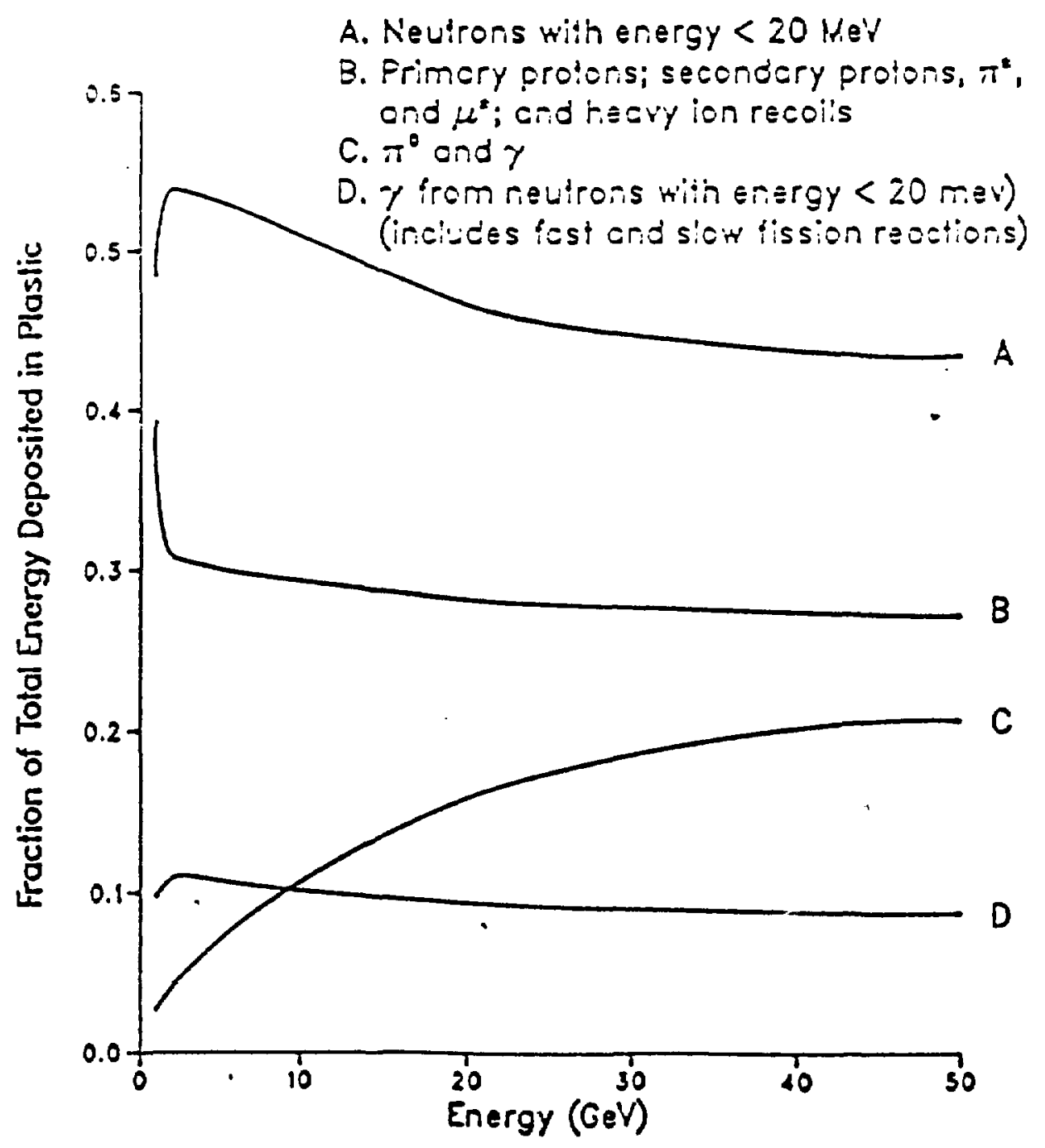

Fig. 17. Fraction of total energy deposited in scintillator located in uranium. 
The hadronic energy resolution from a U/plastic calorimeter is currently the best availab!e.

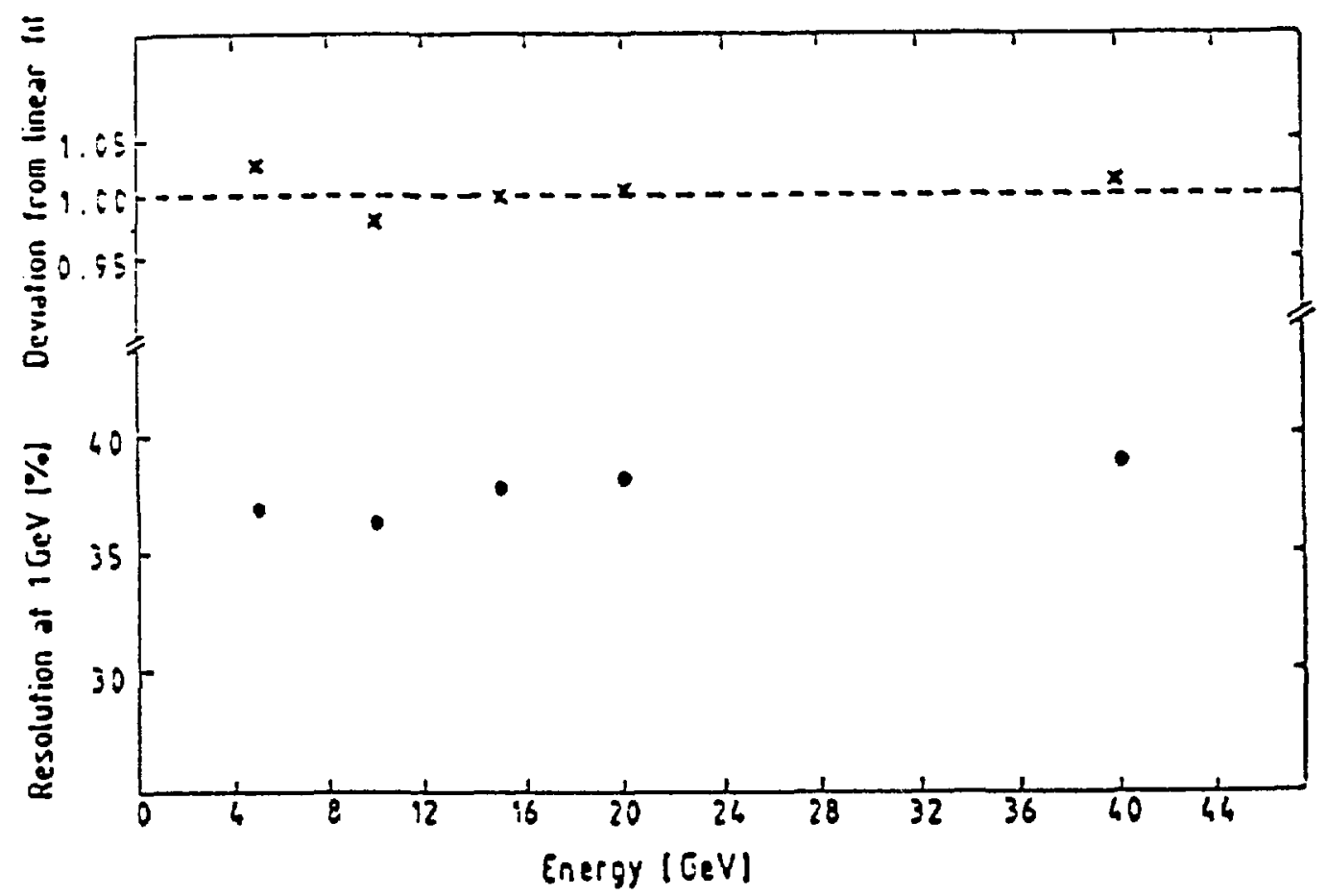

Linearity and Resolution $[\sigma(E) / \sqrt{E}]$ of the Calorimeter for Hadrons. Front Calorimeter U, $2.0 \mathrm{~mm}$, Plastic $2.5 \mathrm{~mm}$, Rear Calorimeter $\quad U, 3.0 \mathrm{~mm}$, Plastic $2.5 \mathrm{~mm}$, 76 unit cells $\mathrm{Cu}, 5.0 \mathrm{~mm}$, Plastic $2.5 \mathrm{~mm}$, 38 unit cells

Fig. 18. Energy resolution of a uranium/copper scintillator calorimeter as a function of energy. 
Compensation is easily achieved with a $\mathrm{U} /$ plastic calorimeter (of proper dimensions, of course).

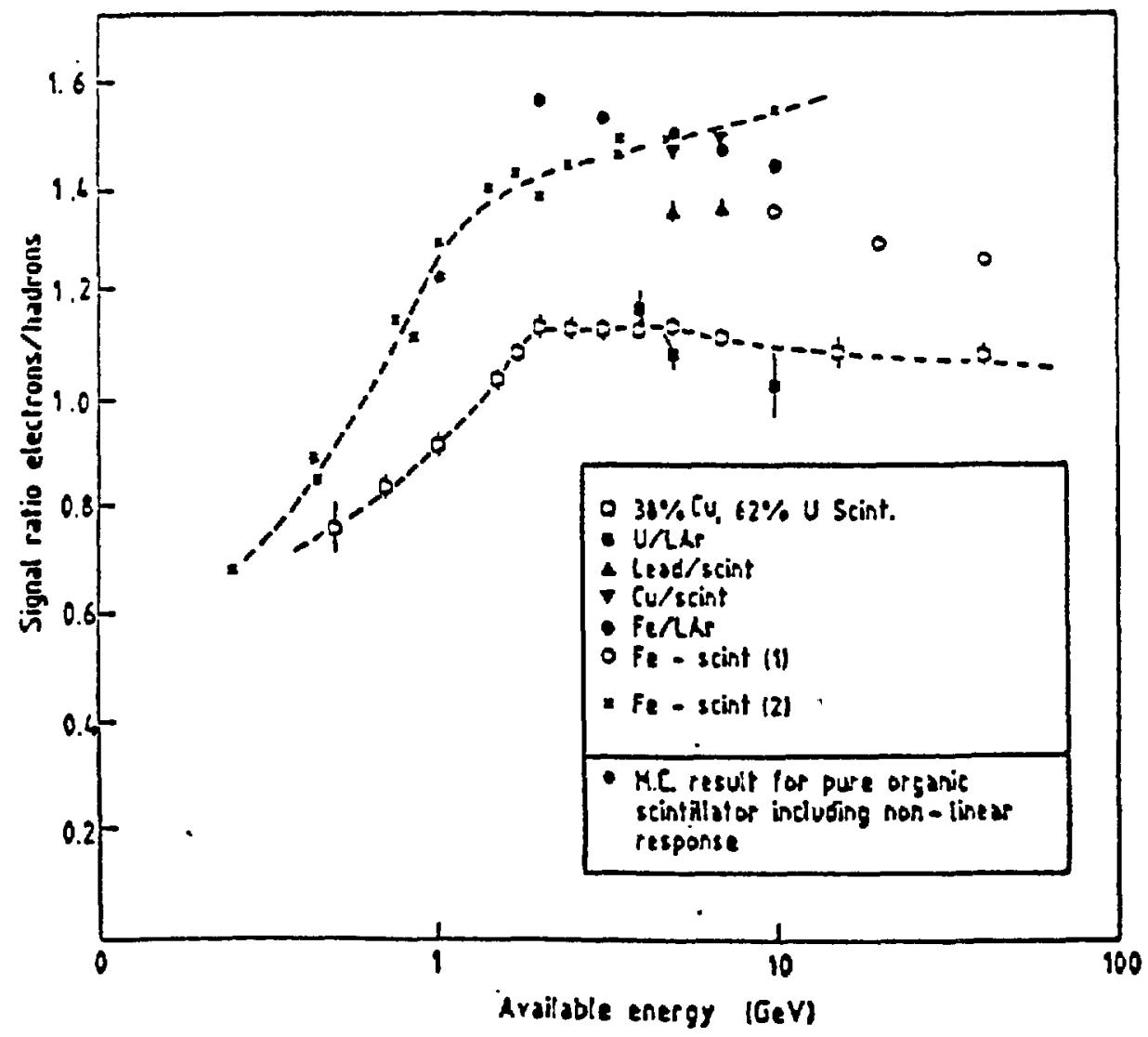

Fig. 19. Compensation characteristics of uranium/copper scintillator and other types of calorimeters. 
Table 6

A Summary of Energy Resolution and Compensation Expected for SSC Detector Types

U/Liq. Ar. U/SI U/IMP Li/Plastic

$\begin{array}{lllll}\mathrm{e} / \mathrm{h} & 1.20-1.05 & 1.15-1.00 & 1.10-0.9 & 1.10-0.9\end{array}$

$(\sigma / E) \sqrt{E}^{*} \quad 45-50 \% \quad 45-50 \% \quad 35-4.5 \%(?) \quad 3.5-10 \%$

(hadrons)

$(\sigma / E) \sqrt{E}$

$-13-17 \%$

(electross)

Assumes fine sampling not necessarily optimum thickness $(5-20 \mathrm{GeV})$. 


\section{SIMULATION PROGRAMS}

How much confidence can be put into the simulation programs such as CALOR89, or GEANT (GHEISHA)? Even though the physics in all of the codes may be good, there is still a large amount of art in the science of simulation. The users of these code systems must rely on the code developers or on those who have extensive exerience using them. For example, the use of a 1.0 $\mathrm{MeV}$ electron/positron cutoff in EGS is almost universal. However, the example given in Fig. 20 illustrates that the Cerenkov signal can be underestimated by as much as $20 \%$ if the cutoff is not set to the Cerenkov cutoff.

The physics in the simulation code must not only be good, but include all important effects. For example, it is possible that two incorrectly included physical effects can cancel each other out and will result in an incorrect explanation of what is really going on inside a calorimetry. This type of situation has happened to the author on two different occasions.

Regardless of the distant past failures of simulation codes, a substantial amount of the understanding of the physics of calorimetry has been obtained from these programs over the years. Some of this understanding includes: electromagnetic sampling inefficiencies, saturation and recombination effects, importance of low energy neutrons, hydrogen, tuning, difficulties with the compensation character of liquid argon, etc.

The current status of simulation codes is basically good. However, improvements are always possible. Whatever degree of accuracy one claims for his particular code, the authors believes the current predictability on energy resolution is $\pm 3 \%$ and on $e / \pi, \pm 5 \%$. Also, general trends can accurately be predicted, but to guarantee that the compensation ratio is 6 to $1 \pm 1 \%$ is beyond even the best of the code systems. 


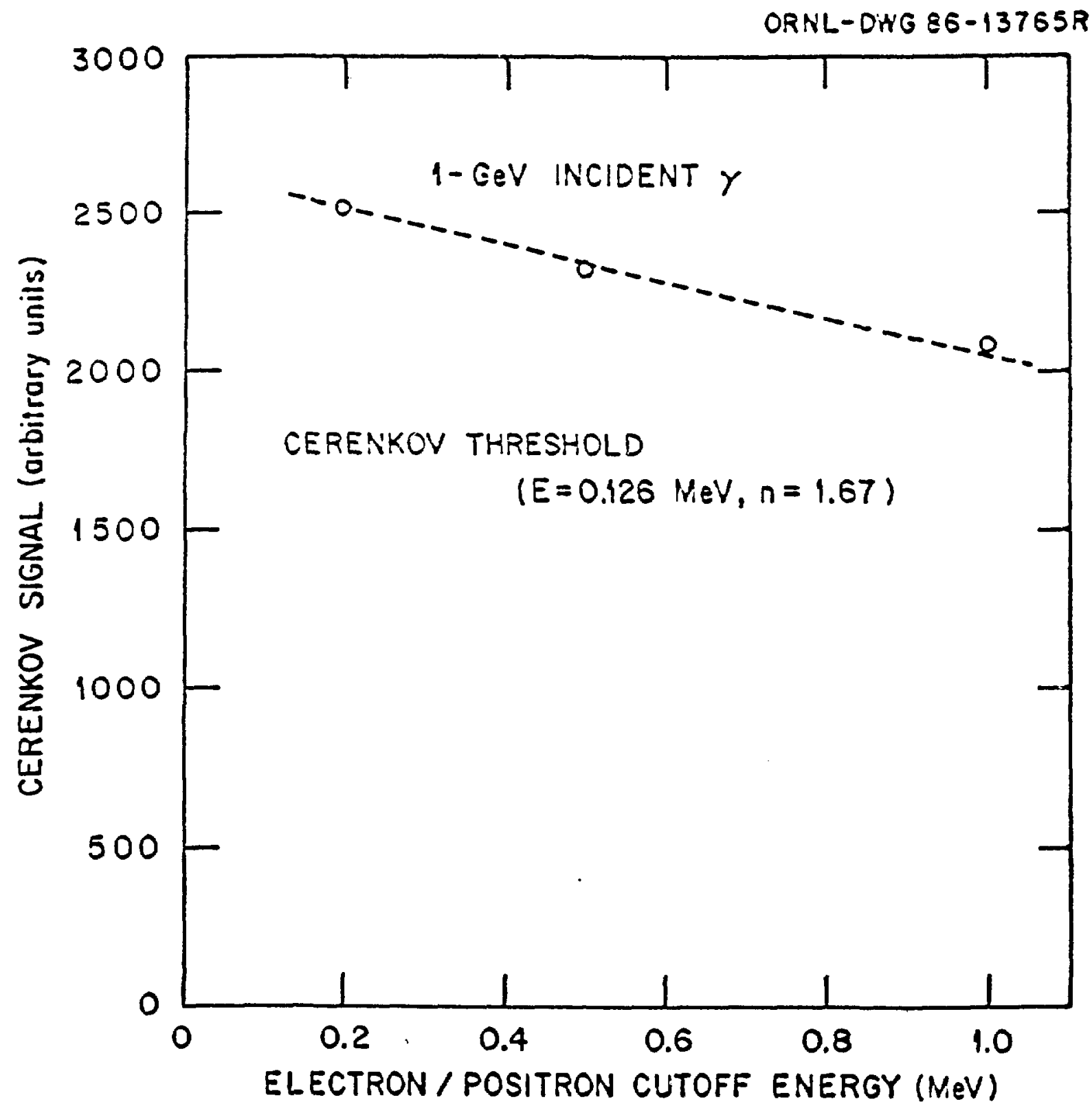

\section{Effect of $\mathrm{e}^{ \pm}$cutoff energy on Cerenkov pulse.}

Fig. 20. The Cerenkov pulse can be substantially underestimated if the correct cutoff energy is not used in the EGS4 code. 


\section{REFERENCES}

1. Fabjan, C. W. et al, Nucl. Instrum. and Methods 141, 61 (1977).

2. Mockett, P. M. Proceedings of the Eleventh SLAC Summer Institute on Particle Physics, July 1983, Ed. Patricia M. McDonough, SLAC-267 (1984).

3. Brau, J. E. Proceedings of the September 1984 Seattle meeting of the SLD Collaboration.

4. Brau, J. E. Proceedings of Workshop on Compensated Calorimetry, CALT68-1305 Pasadena, CA (1985).

5. Gabriel, T. A. et al., "CALOR87: HETC87, MICAP, EGS4, and SPECT, A Code System for Analyzing Detectors for Use in High Energy Physics Experiments," Proceedings of the Workshop on Detector Simulation for the SSC, Argonne National Laboratory, August 1987.

6. Gabriel, T. A. et al., "CALOR89: A Monte Carlo Program Pachage for the Design and Analysis of Calorimeter Systems," Oak Ridge National Laboratory, ORNL/TM-11185, in preparation.

7. Brückmann, H. Proceedings of ITorkshop on Compensated Calorimetry, CALT-68-1305 Pasadena, CA (1985).

8. Knoll, G. Radiation Detection and Measurement, John Wiley and Sons, New York (1979), p. 570.

9. Birks, J. Proc. Phys. Soc. A64, 874 (1951).

10. Hofmann, W. et al, Nucl. Instrum. Methods 135, 151 (1976).

11. Brau, James E. et al., "Prospects for and Tests of Hadron Calorimetry with Silicon," Oak Ridge National Laboratory, ORNL/TM-10954 (1989).

12. Hubbell, J. H. Int. J. Appl. Radiat. Isot. $\underline{33}, 1269$ (1982); Hubbell, J. H., Gimm, H. A. and Orerbo, I. J. Phys. Chem. Ref. Data 9, 1023 (1980);

Storm, E. and Israel, H. I., Nucl. Data Tables $\underline{A 7}, 565$ (1970)

13. Ford, R. L. and Nelson, W. R., Stanford Linear Accelerator Center, Stanford, CA, SLAC-0210 (1978).

14. Bernardi, E. et al, Nucl. Instrum. and Methods A262, 229 (1987).

15. Goodman, M. S. et al., ORNL/TM-10904, December 1988.

16. Albrow, M. G. et al., Nucl. Instrum. Methods A265 (1988).

17. Radermacher, E. CERN-EP/89-01 (1989).

18. Groom, D. (ed.), SSC-SR-1033 (1988).

19. Wilkins, B. D., Fluss, M. J., Kaufman, S. B., Gross, C. E. and Steinberg, E. P., Nucl. Instrum. Methods $\underline{92}, 381$ (1971).

20. Sattler, A. R., Phys. Rev. 138, A1815 (1965).

21 . It is emphasized that the $e / h$ ratio was calculated assuming no saturation in the silicon.

22. Brau, J. E. and Gabriel, T. A., International Conference on Advanced Technology and Particle Physics, Villa Olmo, Lake Como, Italy, June, 1988 (to be published in Nuclear Instruments and Methods).

23. Brau, J. and Gabriel, T. A., Nucl. Instrum. Methods A238, 489 (1985).

24. Gabriel, T. A., Johnson, J. O. and Brau, J. E. Proceedings of the Workshop on Detector Simulation for the SSC, Argonne National Laboratory, August, 1987.

25. Gilchriese, M. G. D., Proceedings of the 1987 SLAC Summer Institute on Particle Physics. 
26. Baltay, C., Huston, J. and Pope, B. G. Proceedings of the 1986 Summer Study on the Physics of the Superconducting Supercollider, Snowmass, Colorado, p. 355 (1986), Eds. R. Donaldson and J. Marx.

27. Aronson, S. et al., Nucl. Instrum. Methods $\underline{4269}$, No. 3 (1988).

28. Akesson, T. et al., Nucl. Instrum. Methods A241, No. 1 (1985).

29. Dr. James Walker, University of Florida, private communication.

\section{ACKNOWLEDGEMENTS}

Thanks are extended to Dr. Jim Brau for his assistance in preparing parts of the text. Also, the author wishes to thank Coralee Zeigler for typing and editing this paper. 